BULLETIN (New Series) OF THE

AMERICAN MATHEMATICAL SOCIETY

Volume 45, Number 3, July 2008, Pages 367-408

S 0273-0979(08)01206-8

Article electronically published on April 8, 2008

\title{
SOME METHODS FOR STUDYING STABILITY IN ISOPERIMETRIC TYPE PROBLEMS
}

\author{
F. MAGGI
}

\begin{abstract}
We review the method of quantitative symmetrization inequalities introduced in Fusco, Maggi and Pratelli, "The sharp quantitative isoperimetric inequality", Ann. of Math.
\end{abstract}

\section{INTRODUCTION}

Our aim is to introduce certain ideas related to the quantitative study of isoperimetric type inequalities. The main efforts are devoted to the proof of a sharp quantitative version of the Euclidean isoperimetric inequality, first proved in FMP1] improving on previous work by Hall, Hayman and Weitsman [HHW, Ha]. The general scheme of proof remains the same, but at different steps it has been possible to simplify the original arguments. Moreover, we have occasionally opted for a rather informal style of presentation, hoping to provide a broadly and easily accessible account of these techniques. There are many relevant variational problems $\mathrm{PS}$, $\mathrm{Ka}$ ] intimately related to the same symmetrization inequalities studied here from a quantitative point of view, and these ideas have already been shown to be flexible enough to be employed in the study of quantitative forms of other variational problems, like sharp Sobolev, Faber-Krahn, isocapacitary, and Cheeger inequalities [FMP2, CFMP1, FMP3]. An even more surprising application is found in the quantitative study of the Gaussian isoperimetric inequality CFMP2, where natural variants of these arguments still play a prominent role. Starting from the seminal paper by Brezis and Lieb [BL], the reader will find various other contributions on quantitative geometric-functional inequalities based on similar or related tools BE, Ci1, Ci2, CEFT, EFT, AFN.

This work covers the material taught in the first part of a Ph.D. course held by the author at the Università di Napoli "Federico II" during May 2007. In the second part of that course we introduced a different approach to the quantitative isoperimetric inequality, where symmetrizations were dropped out in favor of a heavier use of transportation maps. This method allows us to attack problems where minimizers have no particular symmetry, such as in the case of the anisotropic isoperimetric inequality (4.4). This point of view is developed in the forthcoming paper FiMP, and therefore it is not presented in here.

Received by the editors August 29, 2007.

2000 Mathematics Subject Classification. Primary 49Q20.

(C)2008 American Mathematical Society Reverts to public domain 28 years from publication 


\section{The ISOPERIMETRIC PROBLEM IN QUANTITATIVE FORM}

The isoperimetric inequality is the analytic expression of a well-known geometric variational principle: among sets of prescribed measure, balls have the least perimeter. If $E$ is a measurable subset of $\mathbb{R}^{n}, n \geq 1$, with Lebesgue measure $|E|$, the isoperimetric inequality takes the form

$$
n|B|^{1 / n}|E|^{(n-1) / n} \leq P(E), \quad \text { whenever }|E|<\infty .
$$

Here $B$ is the Euclidean unit ball and $P(E)$ denotes the distributional perimeter of $E$. This functional $P:\left\{\right.$ measurable sets of $\left.\mathbb{R}^{n}\right\} \rightarrow[0, \infty]$, discussed in more detail in the next section, enjoys the following basic properties (that do not characterize P) 1

(i) Translation invariance: $P\left(x_{0}+E\right)=P(E)$, for every $x_{0} \in \mathbb{R}^{n}$;

(ii) Scaling law: $P(\lambda E)=\lambda^{n-1} P(E)$, for every $\lambda>0$;

(iii) Extension property: $P(E)$ agrees with the elementary notion of perimeter whenever $E$ is a set with a smooth or polyhedral boundary.

We let $B(x, r)$ denote the ball of radius $r$ and center $x \in \mathbb{R}^{n}$. Then properties (i)(iii) imply that $P(B(x, r))=n|B| r^{n-1}$. If $|B(x, r)|=|E|$, then $r=(|E| /|B|)^{1 / n}$, and the isoperimetric principle $P(B(x, r)) \leq P(E)$ leads to the inequality (1.1).

The isoperimetric inequality holds with strict sign unless the set $E$ is a ball, as shown in Section 3 . This property motivates the study of quantitative forms of (1.1). More precisely, we introduce the isoperimetric deficit of a set $E$ of positive, finite measure, defined as

$$
\delta(E):=\frac{P(E)}{n|B|^{1 / n}|E|^{(n-1) / n}}-1 .
$$

The map $E \mapsto \delta(E)$ defines a scale invariant, translation invariant functional; by the isoperimetric inequality (1.1), we always have $\delta(E) \geq 0$; furthermore $\delta(E)=0$ if and only if $E$ is (up to a null set) a ball, by the characterization of the equality cases in (1.1). Therefore the size of the isoperimetric deficit measures in some way how far a set is from being a ball.

The quantitative problem amounts to relating the size of the isoperimetric deficit to a more explicit notion of distance from the set of all balls. The methods we shall discuss in here have been developed around the notion of Fraenkel asymmetry of $E$, defined as

$$
A(E):=\inf \left\{\frac{|E \Delta B(x, r)|}{|E|}:|B(x, r)|=|E|, x \in \mathbb{R}^{n}\right\} .
$$

This is a nonnegative, translation and scale invariant functional on the family of sets $E$ with positive, finite measure. Furthermore, $A(E)=0$ if and only if $E$ is equivalent to a ball. We seek for control of $A$ in terms of $\delta$. The best result that can be derived, and that we shall prove, is the following theorem (for a brief account on the origins of this result, see Section [5):

Theorem 1.1. There exists a constant $C(n)$ such that

$$
A(E) \leq C(n) \delta(E)^{1 / 2}
$$

\footnotetext{
${ }^{1}$ For example, $E \mapsto \mathcal{H}^{n-1}(\partial E)$ certainly satisfies (i)-(iii), but $\mathcal{H}^{n-1}(\partial E) \geq P(E)$, with strict sign on sufficiently irregular sets; see Section 2.3
} 
for every set $E$ with $0<|E|<+\infty$; equivalently

$$
n|B|^{1 / n}|E|^{(n-1) / n}\left\{1+\frac{A(E)^{2}}{C(n)}\right\} \leq P(E) .
$$

The main goal of these lectures is to present various ideas that reveal useful in proving Theorem 1.1 and to show how these ideas can be used with success in dealing with other related stability problems. The theory of sets of finite perimeter, introduced in the next section, provides a suitable mathematical framework to discuss these matters.

\section{Sets of Finite PERIMEter}

In this section we briefly recall some basic facts about sets of finite perimeter. While the methods employed in proving Theorem 1.1 can be understood without any particular knowledge of this theory, it is also true that rigorous justification of our arguments is better provided in this framework. Therefore, we need at least to introduce the terminology and to heuristically explain some basic facts. In doing this, we essentially follow the original papers of De Giorgi DG1, DG2. For more complete and modern accounts, we refer the reader to $\mathrm{AFP}$ and $\mathrm{EG}$.

Given a set $E \subset \mathbb{R}^{n}$, its characteristic function $1_{E}$ belongs to $L_{\text {loc }}^{1}\left(\mathbb{R}^{n}\right)$. Let us consider the $\varepsilon$-regularization $1_{E, \varepsilon}$ of $1_{E}$. More precisely, we consider a regularizing kernel $\rho$; i.e. we assume $\rho \in C_{c}^{\infty}(B), \rho \geq 0, \int_{\mathbb{R}^{n}} \rho=1$, and define

$$
1_{E, \varepsilon}(x):=\int_{\mathbb{R}^{n}} \rho_{\varepsilon}(x-y) 1_{E}(y) d y, \quad \rho_{\varepsilon}(z):=\frac{1}{\varepsilon^{n}} \rho\left(\frac{z}{\varepsilon}\right),
$$

for $x, z \in \mathbb{R}^{n}$. We now look at the graph of $1_{E, \varepsilon}$, that of course defines a function of class $C^{\infty}\left(\mathbb{R}^{n}\right)$ with values in $[0,1]$. As $\operatorname{spt} \rho_{\varepsilon} \subset \varepsilon B$, we have

$$
1_{E, \varepsilon}(x)= \begin{cases}1, & \text { if }\left|B(x, \varepsilon) \cap\left(\mathbb{R}^{n} \backslash E\right)\right|=0, \\ 0, & \text { if }|B(x, \varepsilon) \cap E|=0 .\end{cases}
$$

When $E$ is a bounded open set with a smooth or polyhedral boundary, and with outer unit normal $\nu_{E}(x)$, we expect

$$
\nabla 1_{E, \varepsilon}\left(x+t \nu_{E}(x)\right) \approx-\frac{\nu_{E}(x)}{\varepsilon}, \quad x \in \partial E,|t|<\varepsilon,
$$

and therefore,

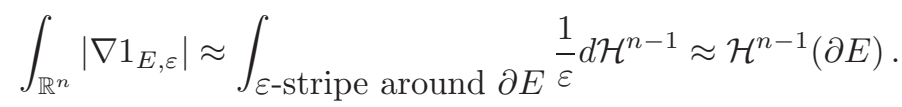

These heuristic remarks motivate the following definition.

Definition. The perimeter $P(E)$ of a set $E$ of $\mathbb{R}^{n}$ is defined as

$$
P(E):=\limsup _{\varepsilon \rightarrow 0^{+}} \int_{\mathbb{R}^{n}}\left|\nabla 1_{E, \varepsilon}\right| .
$$

We say $E$ is a set of finite perimeter if $P(E)<\infty$.

\footnotetext{
${ }^{2}$ All the sets we consider are Borel sets.
} 
2.1. Lower semicontinuity and Gauss-Green measures. The distributional derivative $D 1_{E}$ of $1_{E}$ is defined at a generic $\varphi \in C_{c}^{1}\left(\mathbb{R}^{n}\right)$ by the duality formula

$$
\left(D 1_{E}, \varphi\right)=\int_{E} \nabla \varphi(x) d x .
$$

If the set $E$ has $C^{1}$ boundary with outer unit normal $\nu_{E} \in C^{0}\left(\partial E, S^{n-1}\right)$, then the classical Gauss-Green formula says that the right-hand side of (2.1) equals the integration of $\varphi$ times $\nu_{E}$ over the boundary of $E$,

$$
\int_{E} \nabla \varphi(x) d x=\int_{\partial E} \varphi(x) \nu_{E}(x) d \mathcal{H}^{n-1}(x) .
$$

Therefore, (2.1) is a generalization of (2.2), albeit a tautological one. When $E$ has a finite perimeter much more can be said. The following result provides a first partial result in this direction:

Theorem 2.1. If $E$ is a set of finite perimeter, then there exists an $\mathbb{R}^{n}$-valued Radon measure $\mu_{E}$, called the Gauss-Green measure of E, such that the following distributional Gauss-Green formula holds true for E:

$$
\int_{E} \nabla f(x) d x=\int_{\mathbb{R}^{n}} f(x) d \mu_{E}(x), \quad \forall f \in C_{c}^{1}\left(\mathbb{R}^{n}\right) .
$$

Furthermore, the perimeter of $E$ equals the total variation of $\mu_{E}$ :

$$
P(E)=\left|\mu_{E}\right|\left(\mathbb{R}^{n}\right) .
$$

If, vice versa, a set $E$ and an $\mathbb{R}^{n}$-valued Radon measure $\mu$ are given so that (2.3) holds true, then $E$ is a set of finite perimeter and $\mu=\mu_{E}$.

As this follows by some standard considerations about convolutions and weak convergence of Radon measure, we omit the proof.

Now let $E$ be a set with $C^{1}$ boundary. Then $\mu:=\left.\nu_{E} d \mathcal{H}^{n-1}\right|_{\partial E}$ is a locally bounded $\mathbb{R}^{n}$-valued Borel measure. If $\mathcal{H}^{n-1}(\partial E)<\infty$, then $\mu$ is a Radon measure satisfying (2.3). By the second part of Theorem 2.1, $E$ is a set of finite perimeter, with

Note that if $C$ is a Borel set, then we find

$$
P(E)=\mathcal{H}^{n-1}(\partial E), \quad \mu_{E}=\left.\nu_{E} d \mathcal{H}^{n-1}\right|_{\partial E} .
$$

$$
\left|\mu_{E}\right|(C)=\mathcal{H}^{n-1}(C \cap \partial E) .
$$

This formula is not valid for a generic set of finite perimeter $E$, as we shall explain later. When $A$ is an open set, it indicates that the total variation of $\mu_{E}$ in $A$

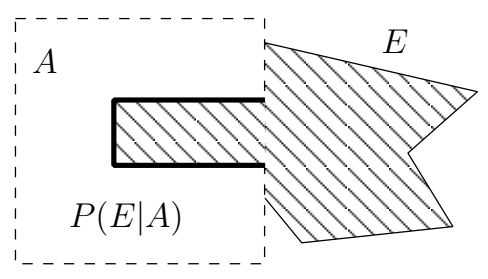

Figure 1. The perimeter of $E$ relative to $A$ is given by that part of the boundary of $E$ lying inside $A$. Parts of the boundary of $E$ that are in common with the boundary of $A$, do not contribute to $P(E \mid A)$. 

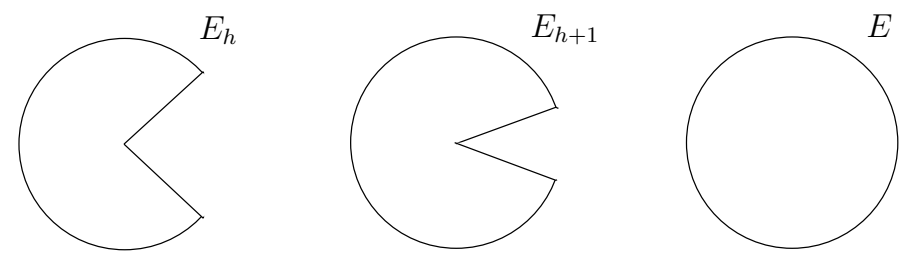

Figure 2 . Here $P(E)=2 \pi, P\left(E_{h}\right) \rightarrow 2 \pi+2$ and $E_{h} \rightarrow E$.

accounts for that fraction of the perimeter of $E$ due to the part of the boundary of $E$ lying inside $A$ (see Figure 1); for this reason $\left|\mu_{E}\right|(A)$ is called the perimeter of $E$ relative to $A$, and is denoted by $P(E \mid A)$. It turns out that the relative perimeter is lower semicontinuous in the $L^{1}$-convergence of measurable sets, according to which $E_{h} \rightarrow E$ if and only if

$$
\left|E_{h} \Delta E\right| \rightarrow 0 \text {. }
$$

In fact we just need to consider the $L^{1}$-local convergence, $E_{h} \rightarrow^{l o c} E$, meaning that $\left|\left(E_{h} \Delta E\right) \cap K\right| \rightarrow 0$ for every compact set $K \subset \mathbb{R}^{n}$.

Theorem 2.2. Let $A$ be open and $E_{h} \rightarrow^{l o c} E$, then

$$
P(E \mid A) \leq \liminf _{h \rightarrow \infty} P\left(E_{h} \mid A\right) .
$$

Proof. Let us recall a basic representation formula for the total variation of an $\mathbb{R}^{n}$-valued measure $\mu$, according to which

$$
|\mu|(A)=\sup \left\{\int_{\mathbb{R}^{n}} \varphi(x) \cdot d \mu(x): \varphi \in C_{c}\left(A, \mathbb{R}^{n}\right),|\varphi(x)| \leq 1\right\},
$$

for every open set $A \subset \mathbb{R}^{n}$. Here we have used the notation

$$
\int_{A} \varphi(x) \cdot d \mu(x)=\sum_{i=1}^{n} \int_{A} \varphi^{(i)}(x) d \mu^{(i)}(x),
$$

where $\varphi^{(i)}$ is the $i$-th component of $\varphi$, and $\mu^{(i)}$ is the $i$-th component measure of $\mu$. If $E$ is a set of finite perimeter and $\mu=\mu_{E}$, then (2.5) implies a representation formula for the relative perimeter. Indeed,

$$
P(E \mid A)=\sup \left\{\int_{E} \operatorname{div} \varphi(x) d x: \varphi \in C_{c}^{1}\left(A, \mathbb{R}^{n}\right),|\varphi(x)| \leq 1\right\},
$$

by the density of $C_{c}^{1}$ in $C_{c}$, thanks to (2.3) and by the definition $P(E \mid A)=\left|\mu_{E}\right|(A)$. The functional $E \mapsto \int_{E} \operatorname{div} \varphi(x) d x$, defined at $\varphi \in C_{c}^{1}\left(A, \mathbb{R}^{n}\right)$ fixed, is continuous with respect to the local $L^{1}$-convergence of sets. Therefore, we achieve the thesis.

Of course the inequality (2.4) can be strict; see Figure 2

2.2. Compactness. It is surprising to see how directly a compactness result for sets of finite perimeter can be derived from the very definition of perimeter.

Theorem 2.3. Let $(X, d)$ be the metric space of Lebesgue measurable sets endowed with the distance $d(E, F)=\left\|1_{E}-1_{F}\right\|_{L^{1}\left(\mathbb{R}^{n}\right)}=|E \Delta F|$. For every $R, p>0$, the set $Y$, defined by

$$
Y=\{E \in X: E \subset R B, P(E) \leq p\},
$$

is compact in $(X, d)$. 
Proof. The argument is based on the elementary Poincaré inequality,

$$
\int_{r Q}\left|f-\frac{1}{r^{n}} \int_{r Q} f\right| \leq \sqrt{n} r \int_{r Q}|\nabla f|, \quad \forall f \in C^{1}(\overline{r Q}),
$$

where $Q=(0,1)^{n}$ is the unit cube of $\mathbb{R}^{n}$. Its simple proof, based on Fubini's Theorem and an induction argument over the dimension $n$, can be found in DG2, Lemma I].

Let us consider the family $\left\{Q_{h}\right\}_{h \in \mathbb{N}}=\left\{r(z+Q): z \in \mathbb{Z}^{n}\right\}$ of disjoint cubes of side length $r$, covering $\mathbb{R}^{n}$ up to a null set, and apply (2.7) to $f=1_{E, \varepsilon}$, so to find

$$
\sqrt{n} r \int_{\mathbb{R}^{n}}\left|\nabla 1_{E, \varepsilon}\right| \geq \sum_{h \in \mathbb{N}} \int_{Q_{h}}\left|1_{E, \varepsilon}-\frac{1}{r^{n}} \int_{Q_{h}} 1_{E, \varepsilon}\right| \text {. }
$$

In the limit $\varepsilon \rightarrow 0,1_{E, \varepsilon} \rightarrow 1_{E}$ in $L_{l o c}^{1}\left(\mathbb{R}^{n}\right)$. By definition of perimeter,

$$
\begin{aligned}
\sqrt{n} r P(E) & \geq \sum_{h \in \mathbb{N}} \int_{Q_{h}}\left|1_{E}-\frac{1}{r^{n}} \int_{Q_{h}} 1_{E}\right|=\sum_{h \in \mathbb{N}} \int_{Q_{h}}\left|1_{E}-\frac{\left|Q_{h} \cap E\right|}{r^{n}}\right| \\
& =\sum_{h \in \mathbb{N}}\left|E \cap Q_{h}\right|\left|1-\frac{\left|Q_{h} \cap E\right|}{r^{n}}\right|+\left|Q_{h} \backslash E\right| \frac{\left|Q_{h} \cap E\right|}{r^{n}} \\
& =\sum_{h \in \mathbb{N}} \frac{\left|E \cap Q_{h}\right|\left|Q_{h} \backslash E\right|}{\left|Q_{h}\right| / 2} .
\end{aligned}
$$

As $|E|<\infty$, there are at most finitely many cubes $Q_{h}$ such that $\left|Q_{h} \cap E\right| \geq\left|Q_{h}\right| / 2$. Up to relabel the indexes, we can assume these are the cubes $Q_{1}, \ldots, Q_{N}$, so that $\left|Q_{h} \backslash E\right| \geq\left|Q_{h}\right| / 2$ if $h \geq N+1$. Let $T=\bigcup_{h=1}^{N} Q_{h}$. Then

$$
\sqrt{n} r P(E) \geq \sum_{h=1}^{N}\left|Q_{h} \backslash E\right|+\sum_{h=N+1}^{\infty}\left|Q_{h} \cap E\right|=|T \backslash E|+|E \backslash T|=d(E, T) .
$$

We now use this construction to prove that $Y$ is totally bounded in $(X, d)$; i.e. for every $\sigma>0$ a finite family $\left\{T_{j}\right\}_{j=1}^{M}$ of elements of $X$ can be found so that $Y$ is covered by the family of the closed $d$-balls of radius $\sigma$ centered at some $T_{j}$. To this end, for a given $\sigma>0$, we define $r$ via the relation $\sqrt{n} r p=\sigma$, and then we consider the corresponding family of cubes $\left\{Q_{h}\right\}_{h \in \mathbb{N}}$ as in the above argument. Then we define $\left\{T_{j}\right\}_{j=1}^{M}$ to be the family of sets obtained as finite unions from $\left\{Q_{h}: Q_{h} \cap R B \neq \emptyset, h \in \mathbb{N}\right\}$. As this last set is finite, $M<\infty$. Furthermore, our construction shows that for every $E \subset R B$, there exists $T \in\left\{T_{j}\right\}_{j=1}^{M}$ such that $d(E, T) \leq \sqrt{n} r P(E)$. As soon as $E \in Y$, and thus $P(E) \leq p$, it follows that $d(E, T) \leq \sigma$.

As $(X, d)$ is a complete metric space and as $Y$ is totally bounded and closed in $(X, d)$, it follows that $Y$ is compact in $(X, d)$.

The compactness and lower semicontinuity theorems proved so far allow us to apply the Direct Method to prove the existence of a minimizer for the following constrained isoperimetric problem:

$$
\inf \{P(E): E \subset R B,|E|=a\},
$$

where $R$ and $a$ satisfy $a<|B| R^{n}$, so that the competition class is nonempty. Indeed, let $m$ denote the infimum, and let $E_{h}$ be a minimizing sequence; i.e. let $P\left(E_{h}\right) \rightarrow m$. By the compactness theorem, up to subsequences, $E_{h} \rightarrow E$, for 
some set $E$. Obviously $|E|=\lim _{h \rightarrow \infty}\left|E_{h}\right|=a$ and $E \subset R B$. Moreover, by lower semicontinuity,

$$
m \leq P(E) \leq \liminf _{h \rightarrow \infty} P\left(E_{h}\right)=m,
$$

and thus $E$ is optimal. Dropping the requirement $E \subset R B$ is not a trivial problem, and requires a more detailed analysis of the isoperimetric problem.

2.3. Approximation by smooth or polyhedral sets. Let us consider the following example. Given a positive sequence $\varepsilon_{h}$ such that $\sum_{h \in \mathbb{N}} \varepsilon_{h}^{n-1}<\infty$, and set $\left\{x_{h}\right\}_{h \in \mathbb{N}}=\mathbb{Q}^{n} \cap[0,1]^{n}$, we let

$$
E=\bigcup_{h \in \mathbb{N}} B\left(x_{h}, \varepsilon_{h}\right) .
$$

The closure of $E$ contains $[0,1]^{n}$, and provided $|E| \leq \sum_{h \in \mathbb{N}} \varepsilon_{h}^{n}<1$, we have $\mathcal{H}^{n-1}(\partial E)=+\infty$. The set $E$, however, has finite perimeter. To see this, let $E_{N}=\bigcup_{h=1}^{N} B\left(x_{h}, \varepsilon_{h}\right)$, so that $E_{N} \rightarrow E$. Then

$$
P(E) \leq \liminf _{N \rightarrow \infty} P\left(E_{N}\right) \leq \liminf _{N \rightarrow \infty} \sum_{h \in \mathbb{N}} P\left(B\left(x_{h}, \varepsilon_{h}\right)\right)<\infty,
$$

as desired. In the last step we have applied the finite subadditivity of the perimeter, that in turn follows from the inequality,

$$
P(E \cup F \mid A)+P(E \cap F \mid A) \leq P(E \mid A)+P(F \mid A),
$$

valid for every open set $A$ and sets of finite perimeter $E$ and $F$. To prove (2.9), it suffices to let $f=1_{E, \varepsilon}, g=1_{F, \varepsilon}$, and note that, on adding up the inequalities

$$
\begin{aligned}
|\nabla(f g)| & \leq g|\nabla f|+f|\nabla g|, \\
|\nabla(f+g-f g)| & \leq(1-g)|\nabla f|+(1-f)|\nabla g|,
\end{aligned}
$$

as $0 \leq f \leq 1,0 \leq g \leq 1$, we find $|\nabla(f g)|+|\nabla(f+g-f g)| \leq|\nabla f|+|\nabla g|$. On integrating over $A$, since $f g \rightarrow 1_{E \cap F}$ and $f+g-f g \rightarrow 1_{E \cup F}$ for $\varepsilon \rightarrow 0$, we come to (2.9).

The above example gives a hint of how very irregular the sets of finite perimeter can be. The theorems we are going to examine in the next two sections are fundamental to handling this complexity. The first result is an approximation theorem.

Theorem 2.4 (Density of smooth or polyhedral sets). If $E$ is a set of finite perimeter, then a sequence $E_{h}$ of bounded open sets with smooth or polyhedral boundary can be found so that $E_{h} \rightarrow E$ and $P\left(E_{h}\right) \rightarrow P(E)$.

The density theorem follows quite directly by the following instance of the Coarea Formula FR.

Theorem 2.5 (Coarea Formula for the total variation). Let $f: \mathbb{R}^{n} \rightarrow \mathbb{R}$ be smooth or piecewise affine, and let $\{f>t\}$ denote the set of points $x$ such that $f(x)>t$. Then

$$
\int_{\mathbb{R}^{n}}|\nabla f|=\int_{\mathbb{R}} P(\{f>t\}) d t .
$$

Proof. For every measurable function $g: \mathbb{R}^{n} \rightarrow \mathbb{R}$, by Fubini's Theorem we have

$$
\int_{\mathbb{R}^{n}} f g=\int_{\mathbb{R}} d t \int_{\{f>t\}} g .
$$


We apply (2.10) to $g=\operatorname{div} v$ for $v \in C_{c}^{1}\left(\mathbb{R}^{n}, B\right)$. We find

$$
-\int_{\mathbb{R}^{n}} \nabla f \cdot v=\int_{\mathbb{R}^{n}} f \operatorname{div} v=\int_{\mathbb{R}} d t \int_{\{f>t\}} \operatorname{div} v \leq \int_{\mathbb{R}} P(\{f>t\}) d t,
$$

thanks to (2.6). At fixed $R>0$, we find a sequence of such $v$ 's approximating (in the $w^{*}-L^{\infty}$ convergence) the bounded measurable vector field

$$
1_{R B}(x) 1_{\left\{y \in \mathbb{R}^{n}: \nabla f(y) \neq 0\right\}}(x) \frac{-\nabla f(x)}{|\nabla f(x)|},
$$

and apply the inequality we have just derived. On passing to the limit, first in the $v$ 's and then as $R \rightarrow \infty$, we deduce that $\int_{\mathbb{R}^{n}}|\nabla f| \leq \int_{\mathbb{R}} P(\{f>t\}) d t$.

To prove the converse inequality, consider the nondecreasing function $m: \mathbb{R} \rightarrow$ $[0, \infty)$ defined by

$$
m(t):=\int_{\{f \leq t\}}|\nabla f| .
$$

By the Lebesgue Theorem on monotone functions, $m^{\prime}(t)$ exists for a.e. $t \in \mathbb{R}$, and satisfies

$$
\int_{\mathbb{R}^{n}}|\nabla f|=m(+\infty)-m(-\infty) \geq \int_{\mathbb{R}} m^{\prime}(t) d t
$$

Let us show that $m^{\prime}(t)=P(\{f>t\})$ for a.e. $t \in \mathbb{R}$. Let

$$
\psi_{\varepsilon}(s):=1_{[t+\varepsilon, \infty)}(s)+\frac{s-t}{\varepsilon} 1_{[t, t+\varepsilon)}(s) .
$$

Then $\int_{\mathbb{R}^{n}} \operatorname{div}\left(\psi_{\varepsilon}(f) v\right)=0$ for every $v \in C_{c}^{1}\left(\mathbb{R}^{n}, B\right)$, and

$$
\int_{\mathbb{R}^{n}} \psi_{\varepsilon}(f) \operatorname{div} v=-\frac{1}{\varepsilon} \int_{\{t+\varepsilon>f>t\}}(\nabla f) \cdot v \leq \frac{1}{\varepsilon} \int_{\{t+\varepsilon>f>t\}}|\nabla f| \leq \frac{m(t+\varepsilon)-m(t)}{\varepsilon} .
$$

As $\varepsilon \rightarrow 0$, one finds that for a.e. $t \in \mathbb{R}$,

$$
\int_{\{f>t\}} \operatorname{div} v \leq m^{\prime}(t) .
$$

The arbitrariness of $v$ together with (2.6) implies the required inequality.

The approximation theorem is then proved as follows.

Proof of Theorem 2.4. Let $f=1_{E, \varepsilon}$, and let $F_{t}=\{f>t\}$. A simple application of Fubini's Theorem shows that

$$
\int_{\mathbb{R}^{n}}\left|1_{E}-f\right|=\int_{0}^{1}\left|E \Delta F_{t}\right| d t
$$

Therefore, for a.e. $t \in(0,1)$, we have $F_{t} \rightarrow E$ as $\varepsilon \rightarrow 0$ and, by lower semicontinuity, $P(E) \leq \liminf _{\varepsilon \rightarrow 0} P\left(F_{t}\right)$. Therefore, Fatou's Lemma implies

$$
P(E) \leq \int_{0}^{1} \liminf _{\varepsilon \rightarrow 0} P\left(F_{t}\right) d t \leq \liminf _{\varepsilon \rightarrow 0} \int_{0}^{1} P\left(F_{t}\right) d t \leq \liminf _{\varepsilon \rightarrow 0} \int_{\mathbb{R}^{n}}|\nabla f|,
$$

where in the last inequality the Coarea Formula has been applied. As the right-hand side converges to $P(E)$, we have proved that, for a.e. $t \in(0,1)$, we have

$$
P(E) \leq \liminf _{\varepsilon \rightarrow 0} P\left(F_{t}\right), \quad P(E)=\int_{0}^{1} \liminf _{\varepsilon \rightarrow 0} P\left(F_{t}\right) d t .
$$

Necessarily, $P(E)=\liminf _{\varepsilon \rightarrow 0} P\left(F_{t}\right)$. According to the Morse-Sard Theorem, at fixed $\varepsilon$ and for a.e. $t \in(0,1)$, the set $F_{t}$ has a smooth boundary. It is then easy to 
construct a sequence $E_{h}$ of sets with smooth boundary such that $\left|E_{h} \Delta E\right| \rightarrow 0$ and $P\left(E_{h}\right) \rightarrow P(E)$.

The above argument actually allows us to approximate $E$ with the level sets of any sequence of functions $f_{h}$ such that $\int_{\mathbb{R}^{n}}\left|1_{E}-f_{h}\right| \rightarrow 0$ and $\int_{\mathbb{R}^{n}}\left|\nabla f_{h}\right| \rightarrow P(E)$. Approaching $1_{E}$ by smooth functions with compact support, one can choose the approximating sets $E_{h}$ to be bounded sets with smooth boundary; using instead compactly supported piecewise affine functions, $E$ is reached by means of bounded sets with polyhedral boundary.

2.4. Density properties and the reduced boundary. As is well known, every measurable set enjoys certain basic density properties that can be summarized by the relation

$$
\lim _{r \rightarrow 0} \frac{|E \cap B(x, r)|}{|B(x, r)|}=1_{E}(x), \quad \text { for a.e. } x \in \mathbb{R}^{n} .
$$

This is often rephrased by saying that a.e. point of $E$ has density one, while a.e. point of $\mathbb{R}^{n} \backslash E$ has density zero, with respect to $E$.

When $E$ has a smooth boundary, one expects that points $x$ belonging to $\partial E$ have density $1 / 2$ : indeed

$$
\frac{|E \cap B(x, r)|}{|B(x, r)|}=\left|B \cap \frac{E-x}{r}\right|
$$

and, if $x \in \partial E$ and $\nu_{E}(x)$ denotes the outer unit normal to $E$, the rescaled sets $(E-x) / r$ are expected to converge (locally) to the negative half-space specified by $\nu_{E}(x)$, namely

$$
\frac{E-x}{r} \stackrel{\operatorname{loc}}{\rightarrow}\left\{y \in \mathbb{R}^{n}: \nu_{E}(x) \cdot(y-x) \leq 0\right\} .
$$

A crucial property of sets of finite perimeter is that this density property of boundary points remains somehow valid, provided the classical notions of boundary point and outer normal are suitably extended.

The Polar Decomposition Theorem for Radon measures applied to the GaussGreen measure $\mu_{E}$ of the set $E$ ensures the existence of a Borel function $\nu_{E}: \mathbb{R}^{n} \rightarrow$ $\mathbb{R}^{n}$, such that $\left|\nu_{E}(x)\right|=1$ for $\left|\mu_{E}\right|$-a.e. $x \in \mathbb{R}^{n}$, and $\mu_{E}=\left|\nu_{E}\right| d \mu_{E}$, i.e.

$$
\int_{\mathbb{R}^{n}} g d \mu_{E}=\int_{\mathbb{R}^{n}} g \nu_{E} d\left|\mu_{E}\right|, \quad \forall g \in C_{c}\left(\mathbb{R}^{n}\right) .
$$

The use of the symbol $\nu_{E}$ is adopted to suggest that $\nu_{E}$ should be regarded 3 as the outer normal to the set of finite perimeter $E$. By the Besicovitch Derivation Theorem for Radon measures, we have that

$$
\nu_{E}(x)=\lim _{r \rightarrow 0} \frac{\mu_{E}(B(x, r))}{\left|\mu_{E}\right|(B(x, r))},
$$

for $\left|\mu_{E}\right|$-a.e. $x \in \mathbb{R}^{n}$.

Definition. The reduced boundary of the set of finite perimeter $E$ is the set $\mathcal{F} E$ of those points $x \in \mathbb{R}^{n}$ such that $\left|\nu_{E}(x)\right|=1$, (2.12) holds true, and

$$
\left|\mu_{E}\right|(B(x, r))>0
$$

for every $r>0$. The Borel vector field $\nu_{E}: \mathcal{F} E \rightarrow S^{n-1}$ is called the measure theoretic outer normal to $E$.

\footnotetext{
${ }^{3}$ And in fact, when $E$ has a smooth or polyhedral boundary, the measure theoretic definition agrees with the classical one.
} 
It turns out that, at points of the reduced boundary $\mathcal{F} E$, the set $E$ behaves much like a smooth set, concerning its density properties.

Theorem 2.6. Let $E$ be a set of finite perimeter. If $x \in \mathcal{F} E$, then

$$
\frac{E-x}{r} \stackrel{\text { loc }}{\longrightarrow}\left\{y \in \mathbb{R}^{n}: \nu_{E}(x) \cdot(y-x) \leq 0\right\} .
$$

Furthermore,

$$
\begin{aligned}
\lim _{\rho \rightarrow 0} \frac{|E \cap B(x, r)|}{|B(x, r)|} & =\frac{1}{2}, \\
\lim _{r \rightarrow 0} \frac{P(E \mid B(x, r))}{r^{n-1} \mathcal{H}^{n-1}\left(B^{n-1}\right)} & =1,
\end{aligned}
$$

where $B^{n-1}=B \cap \mathbb{R}^{n-1}$. The set of points of density $1 / 2$, i.e. the set of those $x \in \mathbb{R}^{n}$ such that (2.13) holds true, is in fact $\mathcal{H}^{n-1}$-equivalent to $\mathcal{F} E$.

The theorem is proved by exploiting the compactness in local convergence of the family of the blown-up sets $(E-x) / r$, as $r \rightarrow 0$. The compactness theorem then gives us a limit set that can be characterized via the Gauss-Green formulas to be the desired half-space. Once the above theorem is proved, an ingenious application of Whitney's Extension Theorem allows us to deduce the following structure result.

Theorem 2.7. Let $E$ be a set of finite perimeter. There are countably many $C^{1}$ hypersurfaces $S_{h}$, compact sets $K_{h} \subset S_{h}$, and a Borel set $N$, such that

$$
\mathcal{F} E=N \cup \bigcup_{h \in \mathbb{N}} K_{h}, \quad \mathcal{H}^{n-1}(N)=0 .
$$

Furthermore, for every $x \in K_{h}$ and every vector $v$ tangent to $S_{h}$ at $x$, we have

$$
\nu_{E}(x) \cdot v=0,
$$

and the Gauss-Green measure $\mu_{E}$ of $E$ is actually related to the $(n-1)$-dimensional Hausdorff measure by

$$
\mu_{E}=\left.\nu_{E} d \mathcal{H}^{n-1}\right|_{\mathcal{F E}}, \quad\left|\mu_{E}\right|=\left.\mathcal{H}^{n-1}\right|_{\mathcal{F E}} .
$$

In particular the Gauss-Green formula

$$
\int_{E} \nabla \varphi(x) d x=\int_{\mathcal{F} E} \varphi(x) \nu_{E}(x) d \mathcal{H}^{n-1}(x),
$$

holds true for every $\varphi \in C_{c}^{1}\left(\mathbb{R}^{n}\right)$.

The first part of Theorem 2.7 should be stated, in more modern terms, by saying that $\mathcal{F} E$ is an $(n-1)$-rectifiable set. We also note that (2.14) is the first instance we met of an analytic formula of geometric content that can be stated in an impressive generality by using the concepts of reduced boundary and measure theoretic outer unit normal of a set $E$.

\section{Steiner SYMmetrization AND the SHARP ISOPERIMETRIC INEQUALITY}

We now turn to the proof of the isoperimetric inequality (1.1) together with a characterization of its equality cases. 


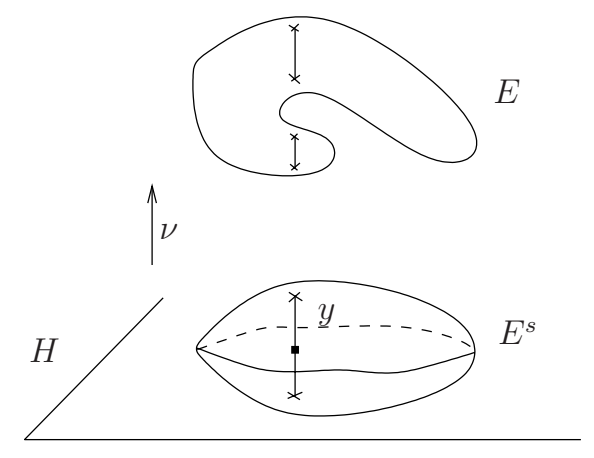

FigurE 3. Steiner symmetrization.

Theorem 3.1 (The sharp isoperimetric inequality). For every measurable set E of finite measure we have

$$
n|B|^{1 / n}|E|^{(n-1) / n} \leq P(E) .
$$

Equality holds if and only if, up to a set of measure zero, E coincides with a ball.

We note that the theorem is in fact trivial if $n=1$. Indeed in this case the conditions $P(E)<\infty$ and $|E|<\infty$ imply that, up to a null set,

$$
E=\bigcup_{i=1}^{N}\left(a_{i}, b_{i}\right), \quad b_{i}<a_{i+1}, \quad N \geq 1 .
$$

Therefore, $\mathcal{F} E=\left\{a_{i}, b_{i}: i=1, \ldots, N\right\}$ and $P(E)=\mathcal{H}^{0}(\mathcal{F} E)=2 N \geq 2$, with equality if and only if $N=1$, i.e. if $E$ is an interval.

The situation is course more delicate when $n \geq 2$. The proof we present here is the one given by De Giorgi in DG3. It is based on the use of the Steiner symmetrization, one of the basic notions of symmetrization for sets, considered in the framework of sets of finite perimeter. A more detailed account on this proof is due to Fusco $[\mathrm{Fs}$, see also $\mathrm{Ta}$.

3.1. Steiner inequality (with some necessary conditions for equality). Given a measurable set $E$, we fix a direction $\nu \in S^{n-1}$ and construct as follows the Steiner symmetral $E^{s}$ of $E$ with respect to $\nu$. Let $H=\left\{y \in \mathbb{R}^{n}: y \cdot \nu=0\right\}$, and let $m: H \rightarrow[0, \infty]$ be defined by

$$
m(y):=\mathcal{H}^{1}(E \cap\{y+t \nu: t \in \mathbb{R}\}), \quad y \in H .
$$

Note that by Fubini's Theorem $m$ is measurable, and in fact $m \in L^{1}\left(\left.d \mathcal{H}^{n-1}\right|_{H}\right)$ as soon as $|E|<\infty$. We set (see Figure 3)

$$
E^{s}=\left\{y+t \nu: y \in H, m(y)>0,-\frac{m(y)}{2} \leq t \leq \frac{m(y)}{2}\right\} .
$$

Thus $E^{s}$ is obtained from $E$ by centering, at every $y \in H$, an interval having the same one-dimensional measure of the vertical section of $E$ over $y$ in the $\nu$ direction. Clearly, $E^{s}$ is a measurable set, symmetric with respect to the hyperplane $H$, and such that $\left|E^{s}\right|=|E|$. The following theorem plays a crucial role in the proof of Theorem 3.1. 
Theorem 3.2 (Steiner inequality). For every set $E$ of finite measure we have

$$
P\left(E^{s}\right) \leq P(E) .
$$

Moreover,

(i) if equality holds, then for $\mathcal{H}^{n-1}$-a.e. $y \in H$ the section $E \cap\{y+t \nu: t \in \mathbb{R}\}$ is $\mathcal{H}^{1}$-equivalent to an interval;

(ii) if $E$ is convex, then equality holds if and only if there exists $c \in \mathbb{R}$ such that $E=E^{s}+c \nu$

The assumption of $E$ having finite measure can be dropped, but we shall not detail this. A full description of the equality cases for the Steiner inequality (3.2) requires a finer analysis; see $\mathrm{CCF}$.

Proof of Theorem 3.2, When $n=1$, then $E^{s}=(-|E| / 2,|E| / 2)$, and the Steiner inequality is the isoperimetric inequality. Therefore, the assertion follows by the simple characterization of sets of finite perimeter in $\mathbb{R}$. Now let $n \geq 2$, and define $m: H \rightarrow[0, \infty]$ as in (3.1). We divide the proof into three steps.

Step one. Let us first examine the case in which $E$ is a bounded set with a polyhedral boundary, whose outer normal field is never orthogonal to the direction of symmetrization $\nu$. Under this assumption, by the Implicit Function Theorem, there exists a partition of $\{m>0\} \subset H$ into finitely many $(n-1)$-dimensional polyhedral sets $C_{1}, \ldots, C_{M}$,

$$
\{y \in H: m(y)>0\}=\bigcup_{j=1}^{M} C_{j}, \quad M \in \mathbb{N},
$$

such that for each $j \in\{1, \ldots, M\}$, there exist finitely many affine functions $g_{j, k}, f_{j, k}$ : $C_{j} \rightarrow \mathbb{R}, k=1, \ldots, N(j)$, satisfying

$$
\begin{aligned}
E \cap\left(C_{j} \times \mathbb{R}\right) & =\left\{y+t \nu: y \in C_{j}, t \in \bigcup_{k=1}^{N(j)}\left(g_{j, k}(y), f_{j, k}(y)\right)\right\}, \\
2 N(j) & =\mathcal{H}^{0}(\mathcal{F} E \cap\{y+t \nu: t \in \mathbb{R}\}), \quad \forall y \in C_{j} .
\end{aligned}
$$

Note that, by construction, if $y \in C_{j}$, then

$$
m(y)=\sum_{k=1}^{N(j)} f_{j, k}(y)-g_{j, k}(y) .
$$

The Steiner inequality is then proved by a simple convexity argument. As $E_{h}$ is bounded by the graphs of $-m / 2$ and $m / 2$, we certainly have

$$
P\left(E^{s}\right)=2 \int_{\{m>0\}} \sqrt{1+\left|\frac{\nabla m}{2}\right|^{2}}=\sum_{j=1}^{M} \int_{C_{j}} \sqrt{4+|\nabla m|^{2}} d \mathcal{H}^{n-1} .
$$


On the other hand,

$$
\begin{aligned}
P(E) & =\sum_{j=1}^{M} \mathcal{H}^{n-1}\left(\mathcal{F} E \cap\left[C_{j} \times \mathbb{R}\right]\right) \\
& =\sum_{j=1}^{M} \int_{C_{j}} \sum_{k=1}^{N(j)} \sqrt{1+\left|\nabla f_{j, k}\right|^{2}}+\sqrt{1+\left|\nabla g_{j, k}\right|^{2}} d \mathcal{H}^{n-1} .
\end{aligned}
$$

On recalling (3.3) and by convexity, we find that at points of $C_{j}$,

$$
\begin{aligned}
& \sum_{k=1}^{N(j)} \sqrt{1+\left|\nabla f_{j, k}\right|^{2}}+\sqrt{1+\left|\nabla g_{j, k}\right|^{2}} \\
\geq & 2 \sum_{k=1}^{N(j)} \sqrt{1+\mid \frac{\nabla f_{j, k}-\left.\nabla g_{j, k}\right|^{2}}{2}=2 N(j)}\left\{\frac{1}{N(j)} \sum_{k=1}^{N(j)} \sqrt{1+\left|\frac{\nabla f_{j, k}-\nabla g_{j, k}}{2}\right|^{2}}\right\} \\
\geq & 2 N(j) \sqrt{1+\left|\frac{1}{N(j)} \sum_{k=1}^{N(j)} \frac{\nabla f_{j, k}-\nabla g_{j, k}}{2}\right|^{2}}=\sqrt{4 N(j)^{2}+|\nabla m|^{2}} .
\end{aligned}
$$

Clearly, $\sqrt{4 N(j)^{2}+|\nabla m|^{2}} \geq \sqrt{4+|\nabla m|^{2}}$, and therefore $P(E) \geq P\left(E^{s}\right)$.

This argument shows in fact that even more is true. Let $D$ be the set of those $y \in H$ such that the vertical section of $E$ over $y$ is not a segment, namely let $D$ be the union of those $C_{j}$ 's such that $N(j) \geq 2$. Then we have

$$
\begin{aligned}
P(E)-P\left(E^{s}\right) & \geq \sum_{j=1}^{M} \int_{C_{j}} \sqrt{4 N(j)^{2}+|\nabla m|^{2}}-\sqrt{4+|\nabla m|^{2}} d \mathcal{H}^{n-1} \\
& =\sum_{j=1}^{M} \int_{C_{j}} \frac{4\left(N(j)^{2}-1\right)}{\sqrt{4 N(j)^{2}+|\nabla m|^{2}}+\sqrt{4+|\nabla m|^{2}}} d \mathcal{H}^{n-1} \\
& \geq 2 \sum_{j=1}^{M} \int_{C_{j} \cap D} \frac{d \mathcal{H}^{n-1}}{\sqrt{4 N(j)^{2}+|\nabla m|^{2}}} .
\end{aligned}
$$

As we have shown that

$$
\sum_{j=1}^{M} \int_{C_{j}} \sqrt{4 N(j)^{2}+|\nabla m|^{2}} \leq P(E),
$$

we easily deduce by the Hölder inequality and the Cauchy-Schwarz inequality that

$$
2 \mathcal{H}^{n-1}(D)^{2} \leq\left(P(E)-P\left(E^{s}\right)\right) P(E) .
$$

Step two. Now let $E$ be a generic set of finite perimeter. By Theorem 2.4 we find a sequence of bounded open sets $E_{h}$ with a polyhedral boundary such that

$$
\left|E_{h} \Delta E\right| \rightarrow 0, \quad P\left(E_{h}\right) \rightarrow P(E) .
$$

As $\nu_{E_{h}}$ takes only finitely many values, up to rotating each $E_{h}$ with a rotation sufficiently close to the identity, we can assume that $\nu_{E_{h}} \cdot \nu \neq 0$ on $\mathcal{F} E_{h}$, without loosing 
(3.5). In this way we ensure that each $E_{h}$ satisfies the assumptions considered in Step one, and therefore we have that

$$
\begin{aligned}
P\left(E_{h}\right) & \geq P\left(E_{h}^{s}\right), \\
2 \mathcal{H}^{n-1}\left(D_{h}\right)^{2} & \leq\left(P\left(E_{h}\right)-P\left(E_{h}^{s}\right)\right) P\left(E_{h}\right),
\end{aligned}
$$

where $D_{h} \subset H$ is the set of those $y \in H$ such that the vertical section of $E_{h}$ over $y$ is not a segment. Let $E_{h}(y)=E_{h} \cap\{y+t \nu: t \in \mathbb{R}\}$ denote this section, and let $E(y)$ be the corresponding section of $E$. Then

$\left|E_{h} \Delta E\right|=\int_{H} \mathcal{H}^{1}\left(E_{h}(y) \Delta E(y)\right) d \mathcal{H}^{n-1}(y) \geq \int_{H}\left|m_{h}(y)-m(y)\right| d \mathcal{H}^{n-1}(y)=\left|E_{h}^{s} \Delta E^{s}\right|$.

In particular $E_{h}^{s} \rightarrow E^{s}$, and by lower semicontinuity, $P\left(E^{s}\right) \leq \liminf _{h \rightarrow \infty} P\left(E_{h}^{s}\right)$. As $P\left(E_{h}\right) \rightarrow P(E)$, by (3.6) we deduce (3.2).

Let us now assume that equality holds in the Steiner inequality for $E$. Then, by carefully passing to the limit in (3.7), we find that

$$
\lim _{h \rightarrow \infty} \mathcal{H}^{n-1}\left(D_{h}\right)=0 .
$$

Recall that $\mathcal{H}^{1}\left(E_{h}(y) \Delta E(y)\right) \rightarrow 0$ for $\mathcal{H}^{n-1}$-a.e. $y \in\{m(y)>0\}$. By the lower semicontinuity of the one-dimensional distributional perimeter, we find that

$$
\liminf _{h \rightarrow \infty} 1_{\left\{m_{h}>0\right\} \backslash D_{h}}(y) \mathcal{H}^{0}\left(\mathcal{F} E_{h}(y)\right) \geq 1_{\{m>0\}}(y) \mathcal{H}^{0}(\mathcal{F} E(y)),
$$

for $\mathcal{H}^{n-1}$-a.e. $y \in H$. By Fatou's Lemma

$$
\begin{aligned}
\int_{\{m>0\}} \mathcal{H}^{0}(\mathcal{F} E(y)) d \mathcal{H}^{n-1}(y) & \leq \liminf _{h \rightarrow \infty} \int_{\left\{m_{h}>0\right\} \backslash D_{h}} \mathcal{H}^{0}\left(\mathcal{F} E_{h}(y)\right) d \mathcal{H}^{n-1}(y) \\
& =2 \mathcal{H}^{n-1}(\{m>0\})
\end{aligned}
$$

By the one-dimensional isoperimetric inequality, $\mathcal{H}^{0}(\mathcal{F} E(y)) \geq 2$. Thus it turns out that $\mathcal{H}^{0}(\mathcal{F} E(y))=2$ for $\mathcal{H}^{n-1}$-a.e. $y \in\{m>0\}$; i.e., at every such $y$, the vertical section of $E$ is $\mathcal{H}^{1}$-equivalent to a segment. This proves (i).

We also note that the arguments presented so far can be easily adapted to show a local version of the Steiner inequality, namely

$$
P(E \mid A \times \mathbb{R}) \geq P\left(E^{s} \mid A \times \mathbb{R}\right), \quad \text { for every open set } A \subset H,
$$

where $A \times \mathbb{R}$ denotes, with an obvious abuse of notation, the set $\{y+t \nu: y \in A$, $t \in \mathbb{R}\}$.

Step three. To conclude the proof of the theorem, let $E$ be convex and such that $P(E)=P\left(E^{s}\right)$. We can find an open convex set $D \subset H$ and pair of concave nonnegative functions $\psi_{1}, \psi_{2}: D \rightarrow[0, \infty)$ such that

$$
\begin{aligned}
E & =\left\{y+t \nu: y \in H,-\psi_{1}(y)<t<\psi_{2}(y)\right\} . \\
E^{s} & =\left\{y+t \nu: y \in H,-\frac{\psi_{1}(y)+\psi_{2}(y)}{2}<t<\frac{\psi_{1}(y)+\psi_{2}(y)}{2}\right\} .
\end{aligned}
$$

Let $D^{\prime}$ be an open set compactly contained in $D$. As $P(E)=P\left(E^{s}\right)$ and (3.8) holds both on $D^{\prime} \times \mathbb{R}$ and $\left(D \backslash D^{\prime}\right) \times \mathbb{R}$, we deduce that

$$
P\left(E \mid D^{\prime} \times \mathbb{R}\right)=P\left(E^{s} \mid D^{\prime} \times \mathbb{R}\right) .
$$


On the other hand, as $\psi_{1}$ and $\psi_{2}$ are locally Lipschitz (their gradient in general will explode on $\partial D$ ), these perimeters can be expressed via the elementary formulas

$$
\begin{aligned}
P\left(E \mid D^{\prime} \times \mathbb{R}\right) & =\int_{D^{\prime}} \sqrt{1+\left|\nabla \psi_{1}\right|^{2}}+\sqrt{1+\left|\nabla \psi_{2}\right|^{2}} d \mathcal{H}^{n-1}, \\
P\left(E^{s} \mid D^{\prime} \times \mathbb{R}\right) & =\int_{D^{\prime}} \sqrt{1+\left|\frac{\nabla \psi_{1}+\nabla \psi_{2}}{2}\right|^{2}} d \mathcal{H}^{n-1} .
\end{aligned}
$$

By strict convexity, there exists $c \in \mathbb{R}$ such that $\psi_{1}-\psi_{2}=c$ on every $D^{\prime}$, i.e. $E=c \nu+E^{s}$.

\subsection{Proof of the isoperimetric variational principle.}

Proof of Theorem 3.1. Let $a, R>0$ with $a<R^{n}|B|$, and consider a minimizer $E$ of the constrained isoperimetric problem (2.8). By the Steiner inequality, if $E$ is optimal in (2.8), then equality holds for $E$ in the Steiner inequality (3.2) with respect to every direction $\nu$.

By (i) in Theorem 3.2. $\mathcal{H}^{n-1}$-a.e. one-dimensional section of $E$ in the direction $\nu$ is $\mathcal{H}^{1}$-equivalent to a segment, and this holds true for every direction $\nu$. Therefore, as pointed out in $[\mathrm{Fs}$, the set $F$ of the Lebesgue points of $E$ is such that every one-dimensional section of $F$ in the direction $\nu$ is a segment, for every direction $\nu$. Thus $F$ is convex.

As $F$ is equivalent to $E$, we have $P(F)=P(E)$ and $|E|=|F|$, thus $F$ is optimal in the constrained isoperimetric problem. Therefore, it is optimal in the Steiner inequality with respect to every direction. By (ii) in Theorem 3.2 , for every $\nu \in S^{n-1}$ we find a constant $c_{\nu} \in \mathbb{R}$ such that

$$
F=c_{\nu} \nu+F_{\nu}^{s},
$$

where $F_{\nu}^{s}$ denotes the Steiner symmetral of $F$ with respect to $\nu$. Thus $F$ is convex and it is symmetric by reflection with respect to every direction, i.e., $F$ is a ball.

Therefore,

$$
\inf \{P(E): E \subset R B,|E|=a\}=P(F)=n|B|^{1 / n} a^{(n-1) / n},
$$

and the infimum is attained if and only if $E$ is equivalent to a ball. In turn this immediately implies that

$$
n|B|^{1 / n}=\inf \left\{\frac{P(E)}{|E|^{(n-1) / n}}: E \text { is bounded }\right\}
$$

with attainment if and only if $E$ is equivalent to a ball. By approximation via bounded sets (Theorem 2.4)

$$
n|B|^{1 / n}=\inf \left\{\frac{P(E)}{|E|^{(n-1) / n}}:|E|<\infty\right\} .
$$

Now let $E$ be an unbounded set with $|E|<\infty$. We have $P(E) \geq n|B|^{1 / n}|E|^{(n-1) / n}$. Assume equality holds: on repeating the above arguments based on the Steiner inequality, $E$ would be equivalent to a ball, against the assumption it is unbounded. Therefore, the sign is always strict on unbounded sets, while in the case of bounded sets we have already characterized the equality cases. 


\section{Small isoperimetric Deficit}

The sharp isoperimetric inequality proved in Theorem 3.1 can be rephrased in terms of the isoperimetric deficit and the Fraenkel asymmetry as follows: whenever $0<|E|<\infty$, then $\delta(E) \geq 0$, with $\delta(E)=0$ if and only if $A(E)=0$. Our main goal is to prove that

$$
A(E) \leq C(n) \delta(E)^{1 / 2} .
$$

This is a good point to make some preliminary remarks on our approach to (4.1). First of all, we shall not make any attempt to evaluate the constant $C(n)$ in front of the deficit. Therefore, here and in the following, $C(n)$ denotes a constant whose value can increase at each instance and depends only from the dimension $n$. We stress that a study of the optimal value of $C(n)$, at present, has been carried over only in dimension $n=2[\mathrm{Bo}$. When dealing with convex sets, an even finer analysis of the two-dimensional case is possible, as shown in $\mathrm{AFN}$.

Next we note that (4.1), in the case $n=1$, is trivial. Indeed if $n=1$, then $\delta(E)=0$ or $\delta(E) \geq 2$; in the first case $A(E)=0$, in the second one it is sufficient to remark that $A(E) \leq 2$ anyway. Therefore, in the following we shall always assume $n \geq 2$.

When $n \geq 2$, inequality (4.1) is sharp in the decay rate of the deficit. This can be seen by a Taylor expansion of the isoperimetric deficit and the Fraenkel asymmetry for the family of axially symmetric ellipsoids

$$
E_{r}=\left\{x \in \mathbb{R}^{n}:\left(r x_{1}\right)^{2}+\sum_{i=2}^{n} x_{i}^{2}=1\right\},
$$

as $r \rightarrow 1$. Another way to check the optimality of the decay rate in (4.1) is to consider a set $E_{\varepsilon}$ obtained by adding onto the top of the ball $B$ a cone of height $\varepsilon$ and radius $r$, with $\varepsilon \ll r \ll 1$. Then, on setting $\omega_{n-1}=\mathcal{H}^{n-1}\left(\mathbb{R}^{n-1} \cap B\right)$,

$$
P\left(E_{\varepsilon}\right) \leq P(B)-\omega_{n-1} r^{n-1}+\omega_{n-1} r^{n-1} \sqrt{1+\frac{\varepsilon^{2}}{r^{2}}}=P(B)+c_{1} \varepsilon^{2}+o\left(\varepsilon^{2}\right),
$$

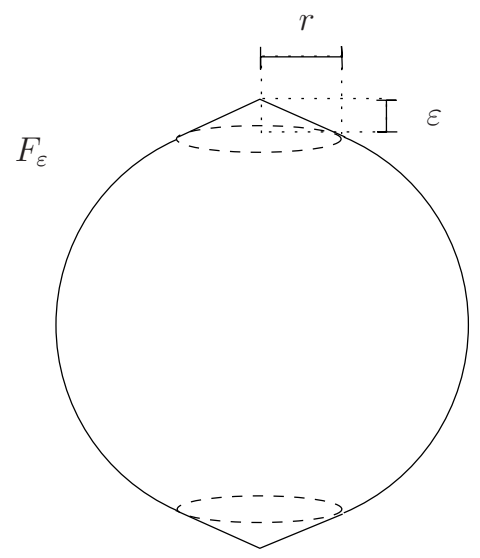

Figure 4 . When $\varepsilon \ll r \ll 1, A\left(F_{\varepsilon}\right)=O(\varepsilon)$ and $\delta\left(F_{\varepsilon}\right)=O\left(\varepsilon^{2}\right)$. 
while $\left|E_{\varepsilon}\right|=|B|+O(\varepsilon)$. Therefore, $\delta\left(E_{\varepsilon}\right) \leq \delta(B)+c_{2} \varepsilon^{2}+o\left(\varepsilon^{2}\right)=O\left(\varepsilon^{2}\right)$. One has to be more careful in estimating the Fraenkel asymmetry, for, if it is clear that $\left|E_{\varepsilon} \Delta B\right|=O(\varepsilon)$, there is the possibility, however, that $A\left(E_{\varepsilon}\right)$ (that is obtained by best approximating $E_{\varepsilon}$ ) is actually of a higher order than $\varepsilon$ as $\varepsilon \rightarrow 0$. To rule out this possibility, one can slightly modify the example as follows. Let $F_{\varepsilon}$ be the set obtained by adding to $B$ two copies of the small cone, centered at opposite poles of $B$. Once again $P\left(F_{\varepsilon}\right)=O\left(\varepsilon^{2}\right)$. On the other hand $F_{\varepsilon}$ is $n$-symmetric with center at the origin. Therefore, as explained in Lemma 5.2 .

$$
A\left(F_{\varepsilon}\right) \leq \frac{\left|F_{\varepsilon} \Delta t_{\varepsilon} B\right|}{\left|F_{\varepsilon}\right|} \leq 3 A\left(F_{\varepsilon}\right), \quad t_{\varepsilon}:=\left(\frac{\left|F_{\varepsilon}\right|}{|B|}\right)^{1 / n} .
$$

As $\left|F_{\varepsilon}\right|=|B|+O(\varepsilon)$, we find that $A\left(F_{\varepsilon}\right)=O(\varepsilon)$.

These introductory remarks well illustrate the point of view we shall adopt in studying (4.1): we are basically working with sets having small isoperimetric deficit. This is soon made rigorous. More precisely, as we are not interested in the constant $C(n)$, we can fix a constant $\delta(n)$ and work directly in the class of sets $E$ such that $\delta(E) \leq \delta(n)$. For, if a constant $K(n)$ has been found so that

$$
A(E) \leq K(n) \delta(E)^{1 / 2}, \quad \text { whenever } \delta(E) \leq \delta(n),
$$

then (4.1) holds on arbitrary sets with

$$
C(n)=\max \left\{K(n), 2 \delta(n)^{-1 / 2}\right\} .
$$

Indeed, as $A(E) \leq 2$, we have that $A(E) \leq C(n) \delta(E)^{1 / 2}$ whenever $\delta(E)>\delta(n)$.

Another recurring idea in the proof of (4.1) is to try to pass from a given class of sets to a narrower class, where the problem of proving (4.1) is hopefully simpler. Roughly speaking, this amounts to showing that given a set $E$ we can find a set $F$ having some good additional properties, like symmetries or something alike, such that the reduction inequalities

$$
A(E) \leq C(n)\left\{A(F)+\delta(E)^{1 / 2}\right\}, \quad \delta(F) \leq C(n) \delta(E),
$$

hold true. Note that the second inequality goes in the natural direction impressed by the requirement that $F$ is "more symmetric" than $E$, while the first one goes opposite; therefore, the two things are essentially in competition.

4.1. Reduction to a priori bounded sets. The following theorem furnishes an illuminating example on the interplay between the small deficit assumption and the process of proving reduction inequalities.

Theorem 4.1. There exists $\delta(n), C(n)$ and $L(n)$ such that for every set $E$ with finite measure and $\delta(E) \leq \delta(n)$ we can find a set $F$, such that $F \subset\left[0,|E|^{1 / n} L(n)\right]^{n}$ and

$$
A(E) \leq C(n)\{A(F)+\delta(F)\}, \quad \delta(F) \leq C(n) \delta(E) .
$$

This result originates from the study of the anisotropic isoperimetric inequality, [EFT, FM]. This is a generalization of the isoperimetric inequality obtained by replacing the Euclidean perimeter with an anisotropic notion of perimeter. Let $K$ be a bounded, open convex set of $\mathbb{R}^{n}$, and let $\|\cdot\|$ be the norm on $\mathbb{R}^{n}$ such that $K$ is the $\|\cdot\|$-unit ball, namely

$$
K:=\left\{x \in \mathbb{R}^{n}:\|x\|<1\right\} .
$$


For every set $E$ of finite perimeter, consider the perimeter functional associated to $K$,

$$
P_{K}(E):=\int_{\mathcal{F} E}\left\|\nu_{E}(x)\right\|_{*} d \mathcal{H}^{n-1}(x),
$$

defined through the dual norm to $\|\cdot\|$,

$$
\|x\|_{*}=\sup \{x \cdot y:\|y\| \leq 1\} .
$$

Then the following variant of the isoperimetric inequality holds true,

$$
n|K|^{1 / n}|E|^{(n-1) / n} \leq P_{K}(E),
$$

and equality holds if and only if $E=x_{0}+r K$ for some $x_{0} \in \mathbb{R}^{n}$ and $r>0$. This inequality, arising from physical considerations concerning the equilibrium of elastic crystals, is particularly challenging from the point of view of the techniques introduced in these notes. Indeed the lack of symmetry of the optimal set $K$ rules out the use of symmetrizations, and a different approach has to be adopted; see EFT, FiMP.

Before coming to the proof of Theorem 4.1 we consider a lemma that turns out to be very useful in proving reduction inequalities such as (4.3).

Lemma 4.2. There exist constants $C(n)$ and $\varepsilon(n)$ such that, when $E$ and $F$ are two sets with

$$
|E \Delta F| \leq \varepsilon|E|, \quad P(F) \leq P(E)+\varepsilon|E|^{(n-1) / n},
$$

for $\varepsilon \leq \varepsilon(n)$, then

$$
A(E) \leq A(F)+4 \varepsilon, \quad \delta(F) \leq \delta(E)+C(n) \varepsilon .
$$

Proof. Let $B_{F}$ be the ball such that $A(F)=\left|F \Delta B_{F}\right| /|F|$, and let $B_{E}$ a ball with the same center as $B_{F}$ and measure equal to $|E|$. Then

$$
\begin{aligned}
A(E)|E| & \leq\left|E \Delta B_{E}\right| \leq|E \Delta F|+\left|F \Delta B_{F}\right|+\left|B_{F} \Delta B_{E}\right| \\
& \leq \varepsilon|E|+A(F)|F|+|| E|-| F|| \leq 2 \varepsilon|E|+A(F)|F|
\end{aligned}
$$

as ||$E|-| F|| \leq|E \Delta F| \leq \varepsilon|E|$. Since $|F| \leq|E|+|E \Delta F|$ and $A(F) \leq 2$, we come to $A(E) \leq A(F)+4 \varepsilon$ (in particular, here $\varepsilon$ does not need to be small). The second inequality comes from a simple Taylor expansion.

Proof of Theorem 4.1. Given $E$ with $\delta(E) \leq \delta(n)$, it suffices to show the existence of a set $F$ such that $F \subset\left[0,|E|^{1 / n} L(n)\right] \times \mathbb{R}^{n-1}$, and

$$
|E \Delta F| \leq C(n) \delta(E)|E|, \quad P(F) \leq P(E)+C(n) \delta(E)|E|^{(n-1) / n} .
$$

Then Lemma 4.2 and an obvious iteration lead to the thesis.

Without loss of generality we can assume that $E$ is bounded with polyhedral boundary and that

$$
\mathcal{H}^{n-1}\left(\left\{x \in \mathcal{F} E: \nu_{E}(x)= \pm e_{1}\right\}\right)=0 .
$$

In this way the function $v(t)=\mathcal{H}^{n-1}\left(E \cap\left\{x_{1}=t\right\}\right)$ is a continuous (see Figure 12), piecewise affine function with compact support $[a, b] \subset \mathbb{R}$, and furthermore

$$
v(t)=|E| \gamma^{\prime}(t), \quad \text { for a.e. } t \in(a, b),
$$

where

$$
\gamma(t):=\frac{\left|E \cap\left\{x_{1}<t\right\}\right|}{|E|} .
$$




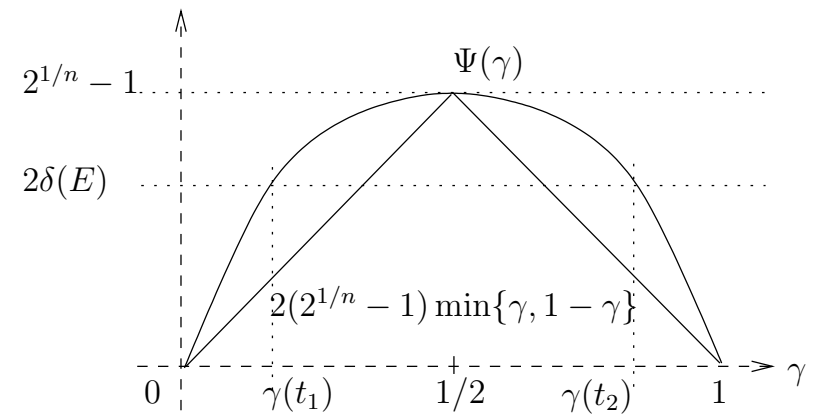

FiguRE 5. The function $\Psi(\gamma)=\gamma^{1 / n^{\prime}}+(1-\gamma)^{1 / n^{\prime}}-1$.

In the small deficit regimen $\delta(E) \leq \delta(n)$, we can provide a lower bound on $v(t)$ in terms of $\gamma(t)$. Indeed, thanks to (4.7) we have $P(E)=P\left(E \mid\left\{x_{1}>t\right\}\right)+$ $P\left(E \mid\left\{x_{1}<t\right\}\right)$, with

$$
\begin{aligned}
& P\left(E \cap\left\{x_{1}<t\right\}\right)=v(t)+P\left(E \mid\left\{x_{1}<t\right\}\right), \\
& P\left(E \cap\left\{x_{1}>t\right\}\right)=v(t)+P\left(E \mid\left\{x_{1}>t\right\}\right) .
\end{aligned}
$$

Therefore, by the isoperimetric inequality (here $n^{\prime}=n /(n-1)$ ),

$$
2 v(t) \geq n|B|^{1 / n}|E|^{1 / n^{\prime}}\left\{\gamma(t)^{1 / n^{\prime}}+(1-\gamma(t))^{1 / n^{\prime}}\right\}-P(E) .
$$

This is most conveniently rewritten in the form

$$
v(t) \geq \frac{n|B|^{1 / n}|E|^{1 / n^{\prime}}}{2}\{\Psi(\gamma(t))-\delta(E)\},
$$

where $\Psi:[0,1] \rightarrow[0,1]$ is the strictly concave function defined by

$$
\Psi(\gamma)=\gamma^{1 / n^{\prime}}+(1-\gamma)^{1 / n^{\prime}}-1 .
$$

Note that $\Psi$ takes its maximum value for $\gamma=1 / 2$, and it is equal to zero at $\gamma=0,1$ (see Figure 5). Therefore,

$$
\Psi(\gamma) \geq 2\left(2^{1 / n}-1\right) \min \{\gamma, 1-\gamma\} .
$$

Then, provided $2 \delta(n)<\Psi(1 / 2)$, we can find $\left(t_{1}, t_{2}\right) \subset(a, b)$ such that

$$
\begin{aligned}
\gamma\left(t_{1}\right) & =1-\gamma\left(t_{2}\right), \\
2 \delta(E) & =\Psi\left(\gamma\left(t_{1}\right)\right)=\Psi\left(\gamma\left(t_{2}\right)\right), \\
\Psi(\gamma(t)) & \geq 2 \delta(E) \quad \forall t \in\left(t_{1}, t_{2}\right) .
\end{aligned}
$$

As a consequence, the following lower bounds hold true for every $t \in\left(t_{1}, t_{2}\right)$ :

$$
\begin{aligned}
& v(t) \geq \frac{n|B|^{1 / n}|E|^{1 / n^{\prime}} \delta(E)}{2}, \\
& v(t) \geq \frac{n|B|^{1 / n}|E|^{1 / n^{\prime}} \Psi(\gamma(t))}{4} .
\end{aligned}
$$




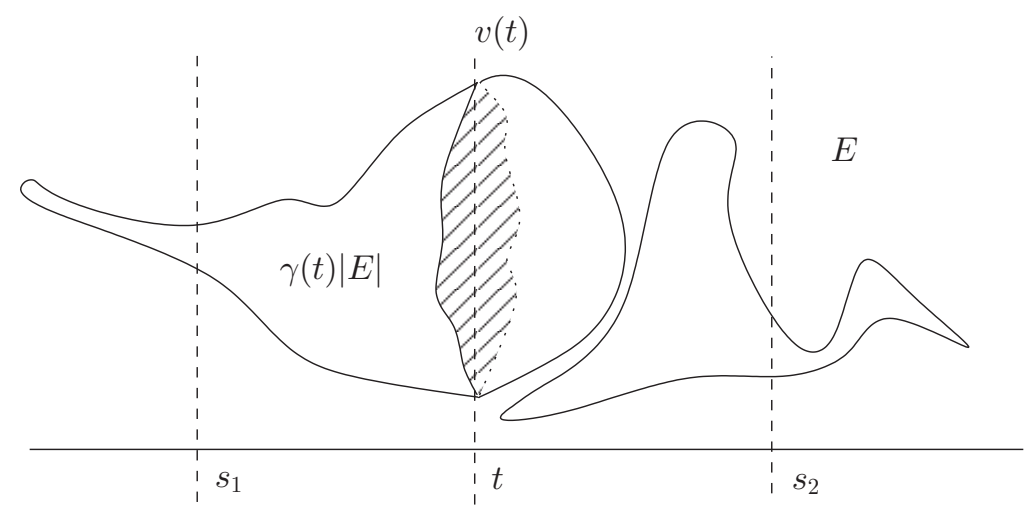

Figure 6. For $t \in\left(s_{1}, s_{2}\right)$, the fraction of volume of $E \cap\left\{x_{1}<t\right\}$ into $E$ is not too close, in terms of $\delta(E)$, to 0 or to 1 ; correspondingly, a lower bound for $v(t)$ in terms of the deficit holds true.

Let $\left(s_{1}, s_{2}\right)$ be the maximal interval such that (4.10) holds; i.e. let

$$
\begin{aligned}
& s_{1}:=\max \left\{t \in\left(a, t_{1}\right]: v(t) \leq \frac{n|B|^{1 / n}|E|^{1 / n^{\prime}} \delta(E)}{2}\right\}, \\
& s_{2}:=\min \left\{t \in\left[t_{2}, b\right): v(t) \leq \frac{n|B|^{1 / n}|E|^{1 / n^{\prime}} \delta(E 0)}{2}\right\} .
\end{aligned}
$$

We claim that $s_{2}-s_{1} \leq L(n)$. Indeed, by (4.9)

$$
\gamma\left(t_{1}\right) \leq \frac{\Psi\left(\gamma\left(t_{1}\right)\right)}{2\left(2^{1 / n}-1\right)}=\frac{\delta(E)}{2^{1 / n}-1},
$$

and therefore, as $\int_{s_{1}}^{t_{1}} v(t) d t=|E|\left(\gamma\left(t_{1}\right)-\gamma\left(s_{1}\right)\right) \leq|E| \gamma\left(t_{1}\right)$,

$$
t_{1}-s_{1} \leq \frac{2}{n|B|^{1 / n}|E|^{1 / n^{\prime}} \delta(E)} \int_{s_{1}}^{t_{1}} v(t) d t=\frac{2|E|^{1 / n} \gamma\left(t_{1}\right)}{n|B|^{1 / n} \delta(E)} \leq \frac{|E|^{1 / n} L(n)}{3} .
$$

One argues similarly in order to estimate $s_{2}-t_{2} \leq|E|^{1 / n} L(n) / 3$, and eventually notes that, thanks to (4.11),

$$
\begin{aligned}
t_{2}-t_{1} & \leq \frac{4}{n|B|^{1 / n}|E|^{1 / n^{\prime}}} \int_{t_{1}}^{t_{2}} \frac{v(t) d t}{\Psi(\gamma(t))}=\frac{4|E|^{1 / n}}{n|B|^{1 / n}} \int_{t_{1}}^{t_{2}} \frac{\gamma^{\prime}(t) d t}{\Psi(\gamma(t))} \\
& =\frac{4|E|^{1 / n}}{n|B|^{1 / n}} \int_{0}^{1} \frac{d \gamma}{\Psi(\gamma)} \leq \frac{|E|^{1 / n} L(n)}{3} .
\end{aligned}
$$

Now let

$$
F=\left[E \cap\left\{x \in \mathbb{R}^{n}: s_{1}<x_{1}<s_{2}\right\}\right]-s_{1} e_{1} .
$$

This set is contained into $\left[0,|E|^{1 / n} L(n)\right] \times \mathbb{R}^{n-1}$; furthermore,

$$
|E \Delta F| \leq|E|\left[\gamma\left(t_{1}\right)+\left(1-\gamma\left(t_{2}\right)\right)\right] \leq C(n)|E| \delta(E) .
$$


Since $v$ is continuous, $v\left(s_{1}\right)=v\left(s_{2}\right)=C(n) \delta(E)|E|^{1 / n^{\prime}}$, and therefore,

$$
\begin{aligned}
P(F) & =P\left(E \mid\left(s_{1}, s_{2}\right) \times \mathbb{R}\right)+\sum_{i=1}^{2} \mathcal{H}^{n-1}\left(E \cap\left\{x_{1}=s_{i}\right\}\right) \\
& \leq P(E)+v\left(s_{1}\right)+v\left(s_{2}\right) \leq P(E)+C(n) \delta(E)|E|^{1 / n^{\prime}} .
\end{aligned}
$$

The theorem is proved.

4.2. A mild quantitative isoperimetric inequality. The following useful theorem comes as an immediate corollary of Theorem 4.1 .

Theorem 4.3. For every $\varepsilon>0$ there exists $\delta(n)>0$ such that $\delta(E) \leq \delta(n)$ implies $A(E) \leq \varepsilon$

Proof. By contradiction. Let $E_{h}$ be a sequence such that $\delta\left(E_{h}\right) \rightarrow 0$ but $\lim _{h \rightarrow \infty} A\left(E_{h}\right)=\varepsilon>0$. Up to a rescaling, $\left|E_{h}\right|=|B|$. Let $F_{h}$ be the set associated to $E_{h}$ by Theorem 4.1. the sequence $F_{h}$ is precompact thanks to Theorem 2.3. If we set $F_{h} \rightarrow F$, then we derive the contradiction $\delta(F)=0$ and $A(F)=\varepsilon>0$ by the lower semicontinuity of the deficit and by the continuity of the Fraenkel asymmetry.

4.3. Reduction to indecomposable sets. In this section we show that, under the small deficit assumption, $E$ can be replaced with a connected set. The argument is a simple variation on the proof of Theorem 4.1.

We start by making precise the sense in which a set of finite perimeter $E$ should be considered connected. A set of finite perimeter $E$ with $0<|E|<\infty$ is said to be indecomposable if

$E=E_{1} \cup E_{2}, \quad E_{1} \cap E_{2}=\emptyset, \quad P(E)=P\left(E_{1}\right)+P\left(E_{2}\right) \quad \Longrightarrow \min \left\{\left|E_{1}\right|,\left|E_{2}\right|\right\}=0$.

Let us recall that every open set of finite measure is the union of countably many disjoint open connected components. In the same spirit, it can be shown (see AFP. Example 4.18]) that every set of finite perimeter $E$ with $0<|E|<\infty$ admits a decomposition

$$
E=\bigcup_{h \in N} E_{h}, \quad \text { with } N \text { a countable set, }
$$

where the $E_{h}$ 's are indecomposable, mutually disjoint sets, such that

$$
P(E)=\sum_{h \in \mathbb{N}} P\left(E_{h}\right) .
$$

We pause now to mention the following simple fact: an optimal set $E$ in the isoperimetric inequality is necessarily indecomposable. Indeed let $E=E_{1} \cup E_{2}$, $E_{1} \cap E_{2}=\emptyset$, and $P(E)=P\left(E_{1}\right)+P\left(E_{2}\right)$. Then

$$
P(E)=P\left(E_{1}\right)+P\left(E_{2}\right) \geq n|B|^{1 / n}\left\{\left|E_{1}\right|^{1 / n^{\prime}}+\left|E_{2}\right|^{1 / n^{\prime}}\right\} \geq n|B|^{1 / n^{\prime}}|E|^{1 / n^{\prime}},
$$

with strict sign, unless $\min \left\{\left|E_{1}\right|,\left|E_{2}\right|\right\}=0$. Therefore, if $E$ is optimal in the isoperimetric inequality, then $E$ is indecomposable. A perturbation of this argument leads to the following result:

Theorem 4.4. There exist $C(n)$ and $\delta(n)$ such that, if $\delta(E) \leq \delta(n)$, then an indecomposable set $F$ can be found such that

$$
A(E) \leq A(F)+C(n) \delta(E), \quad \delta(F) \leq C(n) \delta(E) .
$$


The following proof was found during discussions with A. Figalli.

Proof. Let $E=\bigcup_{h \in N} E_{h}$ be the above-mentioned decomposition of $E$ in indecomposable sets, and let $|E|=|B|$. By concavity,

$$
P(E)=\sum_{h \in N} P\left(E_{h}\right) \geq n|B|^{1 / n} \sum_{h \in N}\left|E_{h}\right|^{1 / n^{\prime}} \geq n|B|^{1 / n}|E|^{1 / n^{\prime}} .
$$

Therefore,

$$
n|B| \delta(E) \geq\left(\sum_{h \in N} a_{h}^{1 / n^{\prime}}\right)-1, \quad a_{h}=\frac{\left|E_{h}\right|}{|E|} .
$$

Let us order the $a_{h}$ 's in a decreasing (possibly finite) sequence. If $a_{1} \geq 1 / 2$, again by concavity, we find that, for a suitable constant $c_{0}=c_{0}(n)$,

$$
n|B| \delta(E) \geq a_{1}^{1 / n^{\prime}}+\left(1-a_{1}\right)^{1 / n^{\prime}}-1 \geq c_{0}\left(1-a_{1}\right)^{1 / n^{\prime}},
$$

i.e. $\left|E \backslash E_{1}\right| \leq C(n) \delta(E)^{n^{\prime}}$. As $P\left(E_{1}\right) \leq P(E)$, we conclude by taking $F=E_{1}$, thanks to Lemma 4.2 .

Let us now show that, if $a_{1}<1 / 2$, then $\delta(E)$ is bounded from below by a positive constant depending only on $n$. Indeed if $a_{1}<1 / 2$, then we can find $M \geq 2$ such that

$$
\sum_{h<M} a_{h}<\frac{1}{2}, \quad \sum_{h \leq M} a_{h} \geq \frac{1}{2} .
$$

The usual argument serves to show that

$$
\begin{aligned}
& n|B| \delta(E) \geq\left(\sum_{h<M} a_{h}\right)^{1 / n^{\prime}}+\left(\sum_{h \geq M} a_{h}\right)^{1 / n^{\prime}}-1 \geq c_{0}\left(\sum_{h<M} a_{h}\right)^{1 / n^{\prime}}, \\
& n|B| \delta(E) \geq\left(\sum_{h \leq M} a_{h}\right)^{1 / n^{\prime}}+\left(\sum_{h>M} a_{h}\right)^{1 / n^{\prime}}-1 \geq c_{0}\left(\sum_{h>M} a_{h}\right)^{1 / n^{\prime}} .
\end{aligned}
$$

Thus we find

$$
C(n) \delta(E)^{n^{\prime}} \geq \sum_{h<M} a_{h}+\sum_{h>M} a_{h}=1-a_{M} \geq 1-a_{1} \geq \frac{1}{2},
$$

as needed.

\section{TWO NOTIONS OF SYMMETRY}

In this section we introduce the two notions of symmetry that play a central role in the proof of Theorem 1.1 and which are given in [FMP1].

We start with a brief and incomplete history of the problem. After previous results in dimension $n=2$ due to Bernstein $\mathrm{Be}$, Bonnesen [Bo] and Osserman Os1, or dealing with the special case of convex sets, as in Fuglede [Fu (where a different notion of asymmetry is in fact of interest), the study of (4.1) has been carried on in full generality by Hall-Hayman-Weitsman [HHW] and Hall [Ha]. On combining the results from these two papers one comes to

$$
A(E) \leq C(n) \delta(E)^{1 / 4} \text {. }
$$

Note that, concerning the decay rate of $A$ with respect to $\delta$, this is a weaker estimate than (4.1). It is instructive to have a closer look at the way (5.1) is proved in 
HHW $\mathrm{Ha}$. The approach is based on symmetrization theory and, more precisely, on the use of Schwarz symmetrization.

5.1. Schwarz symmetrization. Given a direction $\nu$ and a measurable set $E$, by Fubini's Theorem the function $v: \mathbb{R} \rightarrow[0, \infty)$

$$
v(t):=\mathcal{H}^{n-1}\left(E \cap\left\{x \in \mathbb{R}^{n}: x \cdot \nu=t\right\}\right)
$$

is measurable, and belongs to $L^{1}(\mathbb{R})$, provided $|E|<\infty$. Let $r(t)$ be the radius of an $(n-1)$-dimensional ball having $(n-1)$-dimensional measure equal to $v(t)$,

$$
r(t)=\left(\frac{v(t)}{\mathcal{H}^{n-1}\left(B \cap \mathbb{R}^{n-1}\right)}\right)^{1 /(n-1)} .
$$

Then the Schwarz symmetral $E^{*}$ of $E$ is defined as

$$
E^{*}=\left\{x \in \mathbb{R}^{n}: r(x \cdot \nu)>0, \quad|x-(x \cdot \nu) \nu|<r(x \cdot \nu)\right\},
$$

i.e., $E^{*}$ is constructed by centering at the point $t \nu$ an $(n-1)$-dimensional ball of radius $r(t)$, oriented orthogonally to $\nu$, and this for every $t$ such that $r(t)>0$ (see Figure 7).

By construction, $E^{*}$ is axially symmetric along $\nu$ (i.e. for every $t \in \mathbb{R}$ the section $E^{*} \cap\{x: x \cdot \nu=t\}$ is an $(n-1)$-dimensional ball), and by Fubini's Theorem it satisfies $\left|E^{*}\right|=|E|$. As in the case of the Steiner symmetrization, the perimeter is lowered on passing from $E$ to $E^{*}$ :

Theorem 5.1 (Schwarz inequality). For every set of finite perimeter $E$ we have

$$
P\left(E^{*}\right) \leq P(E) .
$$

We shall comment later on the proof of the Schwarz inequality; see Section 7.1 For the moment we limit ourselves to sketching the strategy developed in the papers $\mathrm{HHW}$, $\mathrm{Ha}$ to prove (5.1), which consists in showing the following results:

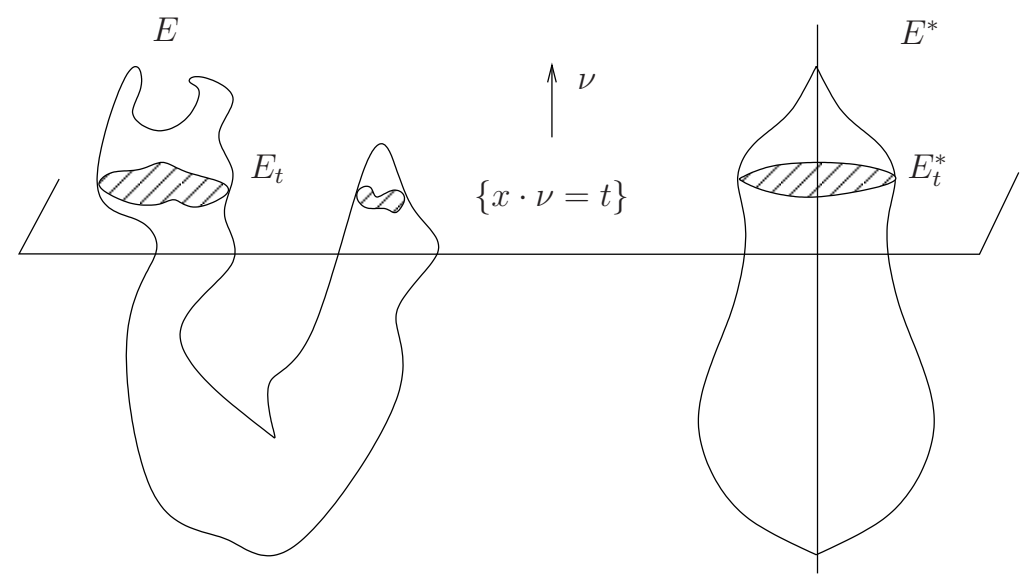

Figure 7. Schwarz symmetrization. The $(n-1)$-dimensional slice $E_{t}=E \cap\{x \cdot \nu=t\}$ of $E$ is rearranged into an $(n-1)$-dimensional ball $E_{t}^{*}=E^{*} \cap\{x \cdot \nu=t\}$, with $\mathcal{H}^{n-1}\left(E_{t}\right)=\mathcal{H}^{n-1}\left(E_{t}^{*}\right)$. 

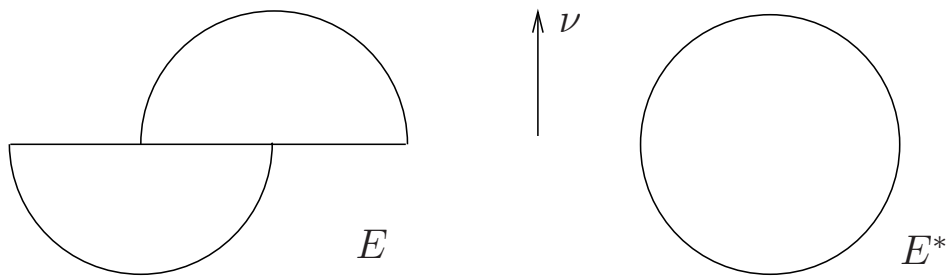

Figure 8. A set $E$, with $A(E)>0$, such that $A\left(E^{*}\right)=0$ for a certain direction $\nu$.

[Ha]: The sharp inequality (4.1) is valid in the class of axially symmetric sets, i.e.

$$
A(E) \leq C(n) \delta(E)^{1 / 2}, \quad \text { whenever } E \text { is axially symmetric . }
$$

This is an easier task than directly showing (4.1) since axially symmetric sets, in practice, are one-dimensional objects.

[HHW] : For every set $E$ we can find a direction $\nu$ such that the corresponding Schwarz symmetral $E^{*}$ satisfies

$$
A(E) \leq C(n) A\left(E^{*}\right)^{1 / 2}, \quad \delta\left(E^{*}\right) \leq \delta(E) .
$$

The second inequality in (5.6) is an immediate consequence of the Schwarz inequality and of $|E|=\left|E^{*}\right|$. The first one requires more attention, as, in general, it can happen that, on randomly picking up $\nu$, we get $A\left(E^{*}\right)=0$ while $A(E)>0$. For example, we can consider

$$
E=[B \cap\{x: x \cdot \nu>0\}] \cup[(y+B) \cap\{x: x \cdot \nu<0\}]
$$

for $y \neq 0$ such that $y \cdot \nu=0$; see Figure 8

By combining the two results, one gets, for a generic set $E, A(E) \leq C(n) A\left(E^{*}\right) \leq$ $C(n) \sqrt{\delta\left(E^{*}\right)} \leq C(n) \delta(E)^{1 / 4}$. Clearly, Hall's part of this proof is sharp, as it is seen once again by looking at the family of ellipsoids considered in Section 4 . However, (5.6) is also optimal (see [HHW]); therefore, there is no possibility of proving (4.1) by directly sharpening these results.

5.2. Reflections by symmetry and $n$-symmetric sets. The proof of (4.1) presented in [FMP1] starts with the idea that in trying to pass directly from a generic set to an axially symmetric set we are making an excessive jump. We rather prefer to introduce an intermediate notion of symmetry, so as to make the reduction process more precise and to preserve the sharp decay rate along the process.

We call $n$-symmetry the notion of symmetry we are interested in: a set $E$ is said to be $n$-symmetric if there are $n$-orthogonal hyperplanes such that $E$ is invariant under reflection with respect to each of them. Alternatively, $E$ is $n$-symmetric if, up to a rigid motion, it is symmetric with respect to the coordinate hyperplanes. The intersection of the $n$ orthogonal hyperplanes of symmetry of $E$ is called the center of symmetry of $E$.

Our proof is then essentially divided into two parts, both using the properties of $n$-symmetric sets in a crucial way. In the first part we show how to reduce the problem (in the spirit of (4.3)) to the class of an $n$-symmetric set. In the second one we show how the reduction to axially symmetric sets can be performed when starting from an $n$-symmetric set without the loss of control met in (5.6). 


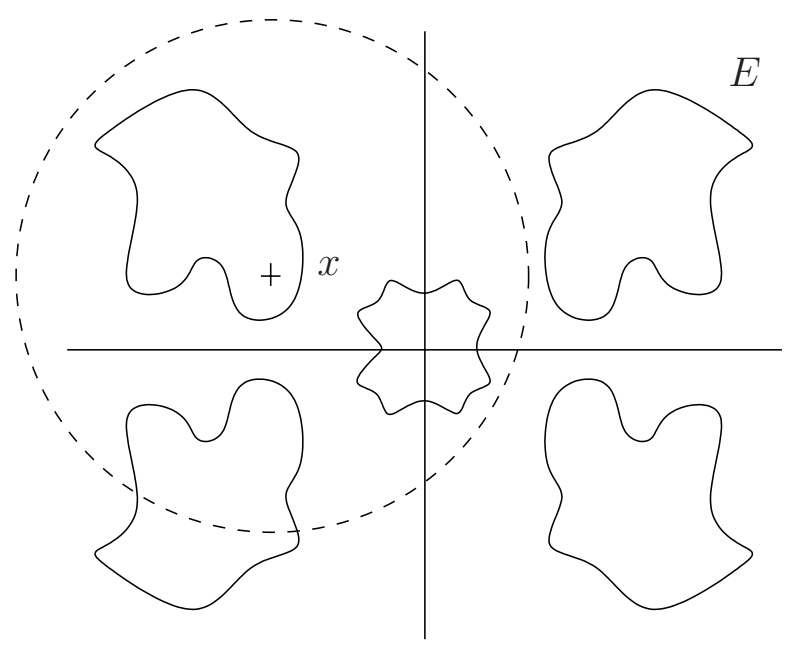

Figure 9. An $n$-symmetric set $E$. The optimal ball for $A(E)$ needs not have the same center of symmetry of $E$.

The next two sections are devoted to the description of these reduction steps. Before going into this, we prove a simple lemma that we shall use many times in the following proofs and which gives a crucial hint on why $n$-symmetry is a relevant property in the study of Fraenkel asymmetry.

Indeed, given a set $E$, there is no way to characterize the position $x$ of the center of the optimal ball $B(x, r)$ realizing $A(E)=|E \Delta B(x, r)| /|E|$. In this respect an $n$-symmetric set offers a simple choice: its center of symmetry. This is not, in general, the optimal point $x$, but we can use it to estimate Fraenkel asymmetry without any loss in the decay rate.

Lemma 5.2. Let $E$ be $n$-symmetric, with center of symmetry at $y \in \mathbb{R}^{n}$. Then

$$
A(E) \leq \frac{|E \Delta B(y, r)|}{|E|} \leq 3 A(E), \quad \text { where } r=(|E| /|B|)^{1 / n} .
$$

Proof. Let $x$ be an optimal center for $E$, i.e. $A(E)=|E \Delta B(x, r)|$, where we are assuming without loss of generality that $|E|=1$. Let $z$ be the reflection of $x$ with respect to $y$. By $n$-symmetry, $z$ is optimal too, i.e. $A(E)=|E \Delta B(z, r)|$; on the other hand it is clear that $|B(x, r) \Delta B(y, r)| \leq|B(x, r) \Delta B(z, r)|$, as $y$ lies in the segment joining $x$ and $z$. Therefore,

$$
\begin{aligned}
A(E) & \leq|E \Delta B(y, r)| \leq|E \Delta B(x, r)|+|B(x, r) \Delta B(y, r)| \\
& \leq|E \Delta B(x, r)|+|B(x, r) \Delta B(z, r)| \\
& \leq 2|E \Delta B(x, r)|+|E \Delta B(z, r)|=3 A(E) .
\end{aligned}
$$

The above argument also proves a more general property. For $k \in\{1, \ldots, n\}$, let $E$ be $k$-symmetric if it is symmetric by reflection with respect to $k$ orthogonal hyperplanes. Furthermore, given a set $F$ in $\mathbb{R}^{n}$, define the relative Fraenkel asymmetry of $E$ with respect to $F$ by

$$
A(E \mid F):=\inf \left\{\frac{|E \Delta B(x, r)|}{|E|}:|B(x, r)|=|E|, x \in F\right\} .
$$


Then we have this lemma:

Lemma 5.3. Let $E$ be a $k$-symmetric set, and let $H$ be the $(n-k)$-dimensional affine subspace of $\mathbb{R}^{n}$ at which the hyperplanes of symmetry of $E$ intersect. Then

$$
A(E) \leq A(E \mid H) \leq 3 A(E)
$$

\section{REDUCTION TO $n$-SYMMETRIC SETS}

The main result proved in this section is the following theorem. Recall that, as usual, $n \geq 2$ and $0<|E|<\infty$.

Theorem 6.1 (n-symmetrization for the isoperimetric inequality). For every set $E$ there exists an $n$-symmetric set $F$ such that the reduction inequalities (4.3) hold true, in the stronger form

$$
A(E) \leq C(n) A(F), \quad \delta(F) \leq C(n) \delta(E) .
$$

The proof is based on a simple notion of symmetrization, that we now pause to discuss.

6.1. Symmetrization by reflection. Given a hyperplane $H$, let us denote by $H^{+}$and $H^{-}$the two corresponding half-spaces in which $\mathbb{R}^{n}$ is divided by $H$, and let $W: \mathbb{R}^{n} \rightarrow \mathbb{R}^{n}$ be the reflection with respect to $H$. Whenever $H$ divides $E$ in equal parts, i.e.

$$
\left|E \cap H^{+}\right|=\left|E \cap H^{-}\right|=\frac{|E|}{2},
$$

we define the symmetrals of $E$ by reflection through $H$ as

$$
\begin{aligned}
& E^{+}=\left[E \cap H^{+}\right] \cup\left[W\left(E \cap H^{+}\right)\right], \\
& E^{-}=\left[E \cap H^{-}\right] \cup\left[W\left(E \cap H^{-}\right)\right] .
\end{aligned}
$$

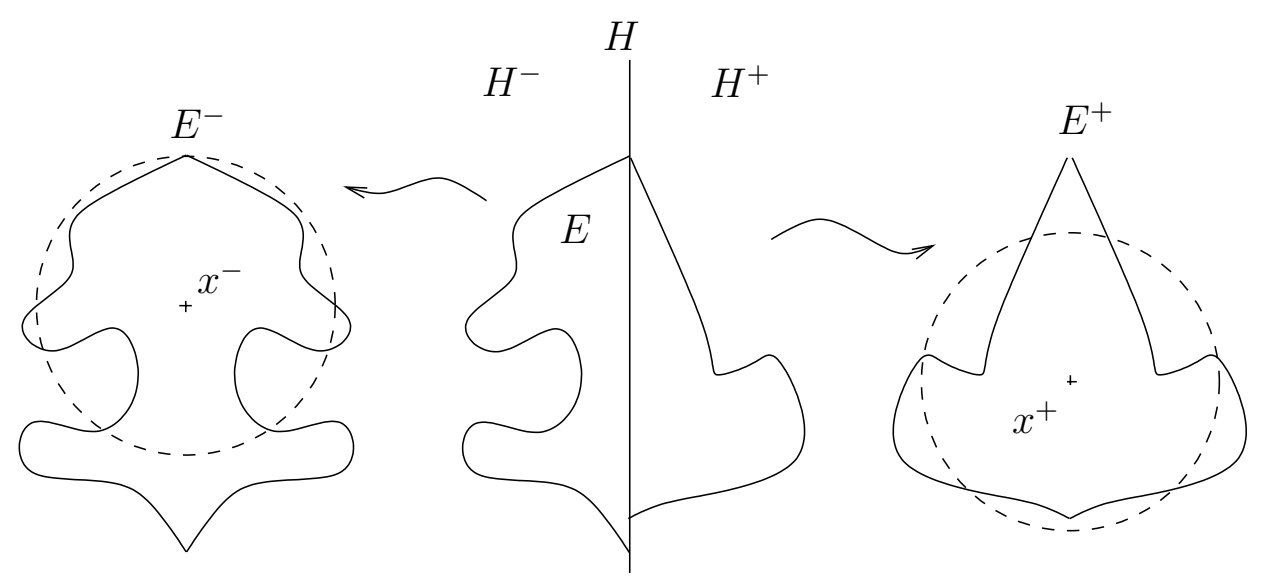

FIGURE 10. Symmetrization by reflection. The strict sign holds in (6.2) for the example in the picture. The centers $x^{+}$and $x^{-}$of the optimal balls for $E^{+}$and $E^{-}$with respect to $H$ need not coincide. 
Clearly, we have $\left|E^{+}\right|=\left|E^{-}\right|=|E|$, and both $E^{+}$and $E^{-}$are symmetric with respect to the hyperplane $H$. The behavior of the perimeter functional with respect to symmetrization by reflection is described by the inequality

$$
\frac{P\left(E^{+}\right)+P\left(E^{-}\right)}{2} \leq P(E) .
$$

Indeed, $P(E)=P\left(E \mid H^{+}\right)+P\left(E \mid H^{-}\right)+\mathcal{H}^{n-1}(\mathcal{F} E \cap H) \geq P\left(E \mid H^{+}\right)+P\left(E \mid H^{-}\right)$, while it is easily seen that

$$
P\left(E^{+}\right)=2 P\left(E \mid H^{+}\right), \quad P\left(E^{-}\right)=2 P\left(E \mid H^{-}\right) .
$$

On dividing by $n|B|^{1 / n}|E|^{(n-1) / n}$ in (6.2), and on taking into account that $\left|E^{+}\right|=$ $\left|E^{-}\right|=|E|$, one immediately comes to

$$
\frac{\delta\left(E^{+}\right)+\delta\left(E^{-}\right)}{2} \leq \delta(E) .
$$

Therefore, up to a factor 2 , the isoperimetric deficit of each reflection symmetral of $E$ is controlled by the isoperimetric deficit of $E$.

In view of proving Theorem 6.1, we are interested in looking at $A\left(E^{+}\right)$and $A\left(E^{-}\right)$compared with $A(E)$. The natural estimate that can be deduced goes as follows. Let $B^{+}$and $B^{-}$denote the optimal balls for $A\left(E^{+} \mid H\right)$ and $A\left(E^{-} \mid H\right)$, respectively. Namely, let

$$
A\left(E^{+} \mid H\right)=\frac{\left|E^{+} \Delta B^{+}\right|}{|E|}, \quad A\left(E^{-} \mid H\right)=\frac{\left|E^{-} \Delta B^{-}\right|}{|E|} .
$$

Then

$$
\begin{aligned}
|E| A(E) & \leq\left|E \Delta B^{+}\right|=\left|\left(E \Delta B^{+}\right) \cap H^{+}\right|+\left|\left(E \Delta B^{+}\right) \cap H^{-}\right| \\
& \leq\left|\left(E \Delta B^{+}\right) \cap H^{+}\right|+\left|\left(E \Delta B^{-}\right) \cap H^{-}\right|+\left|\left(B^{+} \Delta B^{-}\right) \cap H^{-}\right| \\
& =\frac{1}{2}\left(|E| A\left(E^{+} \mid H\right)+|E| A\left(E^{-} \mid H\right)+\left|B^{+} \Delta B^{-}\right|\right),
\end{aligned}
$$

i.e.

$$
A(E) \leq 3\left(A\left(E^{+}\right)+A\left(E^{-}\right)\right)+\frac{\left|B^{+} \Delta B^{-}\right|}{|E|} .
$$

This is essentially the best that can be done, as it may happen that $A\left(E^{+}\right)=$ $A\left(E^{-}\right)=0$, while $A(E)>0$. The typical example is obtained by considering a pair of half-balls mutually shifted along the reflection hyperplane $H$. Assuming that $0 \in H$, we could consider for some $x \in H$,

$$
E=\left[B \cap H^{+}\right] \cup\left[(x+B) \cap H^{-}\right] .
$$

Then clearly $E^{+}=B, E^{-}=x+B$, so that $A\left(E^{+}\right)=A\left(E^{-}\right)=0$; however, $A(E)>0$ (this is the same situation represented in Figure 8). Thus it is impossible to perform a reduction to a 1-symmetric set in the spirit of (6.1) just by picking up a random hyperplane dividing $E$ in equal parts and then selecting one between $E^{+}$and $E^{-}$. The simple idea we shall use to overcome this difficulty is that if a hyperplane is so unlucky that a situation like (6.5) is met, then any orthogonal hyperplane dividing $E$ in equal parts gives a good pair of symmetrals. 


\subsection{Proof of the $n$-symmetrization theorem.}

Proof of Theorem 6.1. Without loss of generality we assume that $|E|=|B|$. We can directly consider the case $\delta(E) \leq \delta(n)$ for a given constant $\delta(n)$. Indeed, let $E_{r}$ be defined as in (4.2), and let $r(n)$ be such that $\delta\left(E_{r(n)}\right)=\delta(n)$. Then for every set $E$ such that $\delta(E)>\delta(n)$, we choose $F=E_{r(n)}$, and find

$$
A(E) \leq 2=\frac{2}{A\left(E_{r(n)}\right)} A(F), \quad \delta(F) \leq \delta(n)<\delta(E) .
$$

As $F$ is $n$-symmetric, we are done. Thus from now on we assume that $\delta(E) \leq \delta(n)$ and $|E|=|B|$. We divide the argument into two steps.

Step one. We show the existence of two constants $C_{0}(n)$ and $\delta_{0}(n)$ such that given $E$ with $|E|=|B|$ and $\delta(E) \leq \delta_{0}(n)$, and given a pair of mutually orthogonal hyperplanes $H_{1}$ and $H_{2}$ dividing $E$ into equal parts, then there is a set $F$, symmetric by reflection with respect to one among $H_{1}$ and $H_{2}$, such that $|E|=|F|$ and the reduction inequalities

$$
A(E) \leq C_{0}(n) A(F), \quad \delta(F) \leq 2 \delta(E),
$$

hold true.

To prove the claim, we consider the symmetrals by reflections of $E$ generated by $H_{1}$ and $H_{2}$, namely

$$
E_{1}^{+}, \quad E_{1}^{-}, \quad E_{2}^{+}, \quad E_{2}^{-} .
$$

By the general properties of symmetrization by reflection, which we have pointed out before, we have automatically that $\delta\left(E_{i}^{\sigma}\right) \leq 2 \delta(E)$. Let $B_{1}^{+}, B_{1}^{-}, B_{2}^{+}, B_{2}^{-}$be the optimal balls for our candidate sets with respect to their hyperplanes of symmetry, namely let

$$
A\left(E_{i}^{\sigma} \mid H_{i}\right)=\frac{\left|E_{i}^{\sigma} \Delta B_{i}^{\sigma}\right|}{|B|}, \quad i \in\{1,2\}, \sigma \in\{+,-\} .
$$

Then from 6.4 we know that

$$
\begin{aligned}
& A(E) \leq 3\left(A\left(E_{1}^{+}\right)+A\left(E_{1}^{-}\right)\right)+\frac{\left|B_{1}^{+} \Delta B_{1}^{-}\right|}{|B|}, \\
& A(E) \leq 3\left(A\left(E_{2}^{+}\right)+A\left(E_{2}^{-}\right)\right)+\frac{\left|B_{2}^{+} \Delta B_{2}^{-}\right|}{|B|} .
\end{aligned}
$$

It suffices to show that, for $\delta(n)$ small enough,

or

$$
\frac{\left|B_{1}^{+} \Delta B_{1}^{-}\right|}{|B|} \leq 17\left[A\left(E_{1}^{+} \mid H_{1}\right)+A\left(E_{1}^{-} \mid H_{1}\right)\right]
$$

$$
\frac{\left|B_{2}^{+} \Delta B_{2}^{-}\right|}{|B|} \leq 17\left[A\left(E_{2}^{+} \mid H_{2}\right)+A\left(E_{2}^{-} \mid H_{2}\right)\right]
$$

and to apply Lemma 5.3 in order to conclude the proof of the claim by selecting, respectively, one between $E_{1}^{+}$and $E_{1}^{-}$, or $E_{2}^{+}$and $E_{2}^{-}$.

Let us argue by contradiction and assume that both the inequalities are false, so that

$$
\begin{aligned}
& \left|E_{1}^{+} \Delta B_{1}^{+}\right|+\left|E_{1}^{-} \Delta B_{1}^{-}\right|<\frac{\left|B_{1}^{+} \Delta B_{1}^{-}\right|}{17}, \\
& \left|E_{2}^{+} \Delta B_{2}^{+}\right|+\left|E_{2}^{-} \Delta B_{2}^{-}\right|<\frac{\left|B_{2}^{+} \Delta B_{2}^{-}\right|}{17} .
\end{aligned}
$$




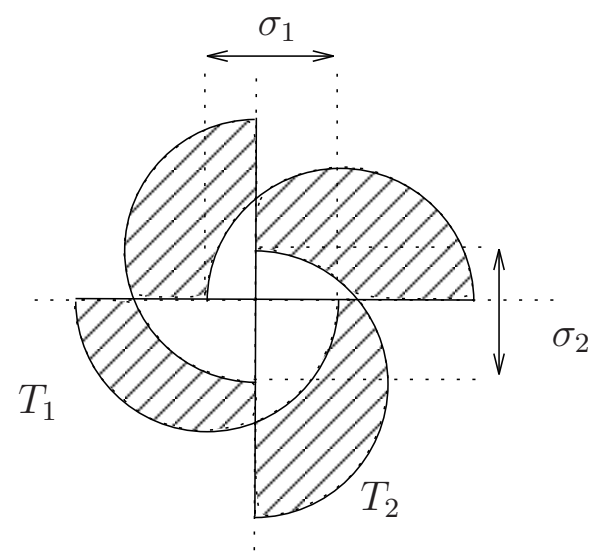

Figure 11. The measure of the symmetric difference of the two pairs of orthogonally shifted half-balls $T_{1}$ and $T_{2}$ controls the distances $\sigma_{1}$ and $\sigma_{2}$ between the centers of $B_{1}^{+}$and $B_{1}^{-}$and of $B_{2}^{+}$ and $B_{2}^{-}$. In particular, $\left|T_{1} \Delta T_{2}\right|$ controls the measures $\left|B_{1}^{+} \Delta B_{1}^{-}\right|$ and $\left|B_{2}^{+} \Delta B_{2}^{-}\right|$.

We now look at the two pairs of orthogonally shifted half-balls $T_{1}$ and $T_{2}$ defined as

$$
\begin{aligned}
& T_{1}=\left(B_{1}^{+} \cap H_{1}^{+}\right) \cup\left(B_{1}^{-} \cap H_{1}^{-}\right), \\
& T_{2}=\left(B_{2}^{+} \cap H_{2}^{+}\right) \cup\left(B_{2}^{-} \cap H_{2}^{-}\right) .
\end{aligned}
$$

Thanks to (6.6) and (6.7), we have

$$
\left|T_{1} \Delta T_{2}\right| \leq\left|T_{1} \Delta E\right|+\left|T_{2} \Delta E\right|=\sum_{i=1}^{2} \frac{\left|E_{i}^{+} \Delta B_{i}^{+}\right|}{2}+\frac{\left|E_{i}^{-} \Delta B_{i}^{-}\right|}{2} \leq \frac{1}{17} \sum_{i=1}^{2} \frac{\left|B_{i}^{+} \Delta B_{i}^{-}\right|}{2} .
$$

We are now going to show that, provided $\delta_{0}(n)$ is small enough,

$$
\max \left\{\left|B_{1}^{+} \Delta B_{1}^{-}\right|,\left|B_{2}^{+} \Delta B_{2}^{-}\right|\right\} \leq 16\left|T_{1} \Delta T_{2}\right| .
$$

The heuristic reason to expect this happens is that $\left|T_{1} \Delta T_{2}\right|$ controls the distances $\sigma_{1}$ and $\sigma_{2}$ between the centers of $B_{1}^{+}$and $B_{1}^{-}$and of $B_{2}^{+}$and $B_{2}^{-}$. In turn, these two quantities control $\left|B_{1}^{+} \Delta B_{1}^{-}\right|$and $\left|B_{2}^{+} \Delta B_{2}^{-}\right|$, as depicted in Figure 11]. Note that by plugging (6.9) into (6.8) one finds

$$
\left|T_{1} \Delta T_{2}\right| \leq \frac{16}{17}\left|T_{1} \Delta T_{2}\right|,
$$

i.e., $T_{1}=T_{2}$. Therefore $\delta(E)=0$, against the strict sign in (6.6) and (6.7). It remains to check the validity of (6.9), which can be justified by the following soft continuity argument based on the small deficit assumption.

As $E_{i}^{\sigma}$ is symmetric with respect to the hyperplane $H_{i}$, we know by Lemma 5.3 that $A\left(E_{i}^{\sigma} \mid H_{i}\right) \leq 3 A\left(E_{i}^{\sigma}\right)$. On the other hand, we also know that $\delta\left(E_{i}^{\sigma}\right) \leq 2 \delta(E)$. Therefore, we can choose $\varepsilon(n)$, and up to taking $\delta_{0}(n)$ small enough, we can work under the assumption that

$$
\max _{i \in\{1,2\}, \sigma \in\{+,-\}}\left\{A(E), A\left(E_{i}^{\sigma} \mid H_{i}\right)\right\} \leq \varepsilon(n),
$$


thanks to Theorem 4.3. In general, the centers of the balls $B_{i}^{\sigma}$ are all different from the center of the optimal ball for $E$; and likewise, though each of the $H_{i}$ 's divides $E$ into equal parts, it will happen that none of the four quarter-spaces

$$
H_{1}^{\sigma} \cap H_{2}^{\tau}, \quad(\sigma, \tau) \in\{+,-\}^{2},
$$

encloses exactly one quarter of the measure of $E$ or of the balls $B_{i}^{\sigma}$. However, provided $\varepsilon(n)$ is small enough, thanks to (6.10), the centers of the various balls $B_{i}^{\sigma}$ shall be as close as wanted to the center of the optimal ball for $E$, and each quarter-space $H_{1}^{\sigma} \cap H_{2}^{\tau}$ shall be as close as wanted to cut $E$ and each of the balls in four equal parts. Therefore, we can choose $\varepsilon(n)$ so small that (6.10) implies

$$
\left|\left(B_{1}^{\sigma} \Delta B_{2}^{\tau}\right) \cap\left(H_{1}^{\sigma} \Delta H_{2}^{\tau}\right)\right| \geq \frac{\left|B_{1}^{\sigma} \Delta B_{2}^{\tau}\right|}{8},
$$

for every $\sigma, \tau \in\{+,-\}$. By (6.11),

$$
\left|T_{1} \Delta T_{2}\right| \geq\left|\left(T_{1} \Delta T_{2}\right) \cap\left(H_{1}^{\sigma} \cap H_{2}^{\tau}\right)\right|=\left|\left(B_{1}^{\sigma} \Delta B_{2}^{\tau}\right) \cap\left(H_{1}^{\sigma} \cap H_{2}^{\tau}\right)\right| \geq \frac{\left|B_{1}^{\sigma} \Delta B_{2}^{\tau}\right|}{8},
$$

and in particular

$$
\left|B_{1}^{+} \Delta B_{1}^{-}\right| \leq\left|B_{1}^{+} \Delta B_{2}^{+}\right|+\left|B_{2}^{+} \Delta B_{1}^{-}\right| \leq 16\left|T_{1} \Delta T_{2}\right| .
$$

Since we have similarly $\left|B_{2}^{+} \Delta B_{2}^{-}\right| \leq 16\left|T_{1} \Delta T_{2}\right|$, (6.9) follows, and the theorem is proved.

Step two. We prove that given any $k \in\{0, \ldots, n-2\}$ and a $k$-symmetric set $F_{k}$ with $\delta\left(F_{k}\right) \leq \delta_{0}(n)$, then a $k+1$ symmetric set $F_{k+1}$ can be found so that $\left|F_{k+1}\right|=\left|F_{k}\right|$ and

$$
A\left(F_{k}\right) \leq C(n) A\left(F_{k+1}\right), \quad \delta\left(F_{k+1}\right) \leq 2 \delta\left(F_{k}\right) .
$$

Indeed, as $k \leq n-2$, we can find two mutually orthogonal hyperplanes $H_{1}$ and $H_{2}$, both dividing $F_{k}$ into equal parts and both orthogonal to the $k$-hyperplanes of symmetry of $F_{k}$. On arguing on $\left(F_{k}\right)_{1}^{+},\left(F_{k}\right)_{1}^{-},\left(F_{k}\right)_{2}^{+},\left(F_{k}\right)_{2}^{-}$as in Step one, we have that by selecting $F_{k+1}$ among those four sets, we certainly have

$$
A\left(F_{k}\right) \leq C(n) A\left(F_{k+1}\right), \quad \delta\left(F_{k+1}\right) \leq 2 \delta\left(F_{k}\right) .
$$

Furthermore, by construction, $F_{k+1}$ is $(k+1)$-symmetric.

By a finite induction argument it is immediately shown that for every $E$ such that $\delta(E) \leq 2^{-n} \delta_{0}(n)$, we can find an $(n-1)$-symmetric set $G$ such that $|E|=|G|$ and

$$
A(E) \leq C(n) A(G), \quad \delta(G) \leq 2^{n-1} \delta(E) .
$$

Now let $H$ be a hyperplane dividing $G$ into equal parts, and let it be orthogonal to the $(n-1)$-hyperplanes of symmetry of $G$. Let us consider the symmetrals by reflection $G^{+}$and $G^{-}$of $G$ with respect to $H$. By construction they are both $n$-symmetric, with $\delta\left(G^{\sigma}\right) \leq 2 \delta(G) \leq 2^{n} \delta(E)$. Furthermore, $G^{+}$and $G^{-}$have the same center of symmetry, so that if $B_{0}$ is the ball of measure $|E|$ centered at that point, we certainly have

$$
\frac{\left|G^{+} \Delta B_{0}\right|}{|E|} \leq 3 A\left(G^{+}\right), \quad \frac{\left|G^{-} \Delta B_{0}\right|}{|E|} \leq 3 A\left(G^{-}\right),
$$


as a consequence of Lemma 5.2 Therefore

$$
\begin{aligned}
A(E) & \leq C(n) A(G) \leq C(n) \frac{\left|G \Delta B_{0}\right|}{|G|}=C(n)\left(\frac{\left|G^{+} \Delta B_{0}\right|+\left|G^{-} \Delta B_{0}\right|}{2|E|}\right) \\
& \leq C(n)\left(A\left(G^{+}\right)+A\left(G^{-}\right)\right) .
\end{aligned}
$$

It suffices to take $F=G^{+}$or $F=G^{-}$to prove the theorem.

\section{The induction ARgument on the Schwarz Inequality}

We eventually come to the proof of Theorem 1.1. Before this we briefly discuss the Schwarz inequality in connection with the Coarea Formula.

7.1. The Schwarz inequality and the Coarea Formula. We now give a sketch of the proof of the Schwarz inequality, based on a particular case of the Coarea Formula for rectifiable sets. We start by setting some notation. We consider a set $E$ of finite measure and set

$$
E_{t}:=E \cap\left\{x: x_{1}=t\right\}, \quad v(t)=\mathcal{H}^{n-1}\left(E_{t}\right) .
$$

As $|E|=\int_{\mathbb{R}} v(t) d t$, we have that $v \in L^{1}(\mathbb{R})$. As we said, the Schwarz symmetral $E^{*}$ of $E$ with respect to the $x_{1}$-axis is given by

$$
E^{*}=\left\{x \in \mathbb{R}^{n}: v\left(x_{1}\right)>0, \quad\left|x-x_{1} e_{1}\right|^{2}<r\left(x_{1}\right)\right\},
$$

where $r(t)=\left(v(t) / \omega_{n-1}\right)^{1 /(n-1)}$ and

$$
\omega_{k}:=\mathcal{H}^{k}\left(B \cap \mathbb{R}^{k}\right), \quad 1 \leq k \leq n .
$$

Our subsequent analysis of $E$ and $E^{*}$ is based on the following result, containing a particular instance of the Coarea Formula for rectifiable sets, and a special case of a result by $\mathrm{Vol}^{\prime}$ pert on slicing sets of finite perimeter.

Theorem 7.1. Let $E$ be a set of finite perimeter in $\mathbb{R}^{n}, n \geq 2$. Then, for a.e. $t \in$ $\mathbb{R}$, the section $E_{t}$ is a set of finite perimeter in $\mathbb{R}^{n-1}$ and its reduced boundary $\mathcal{F} E_{t}$ is $\mathcal{H}^{n-2}$-equivalent to the $t$-section $(\mathcal{F} E)_{t}$ of the reduced boundary of $E$. Furthermore, for every Borel function $g: \mathbb{R}^{n} \rightarrow[0, \infty]$ we have

$$
\int_{\mathcal{F} E} g \sqrt{1-\left|\nu_{E} \cdot e_{1}\right|^{2}} d \mathcal{H}^{n-1}=\int_{\mathbb{R}} d t \int_{(\mathcal{F} E)_{t}} g(t, z) d \mathcal{H}^{n-2}(z),
$$

where $\nu_{E} \cdot e_{1}$ denotes the first component of the outer unit normal vector to $E$.
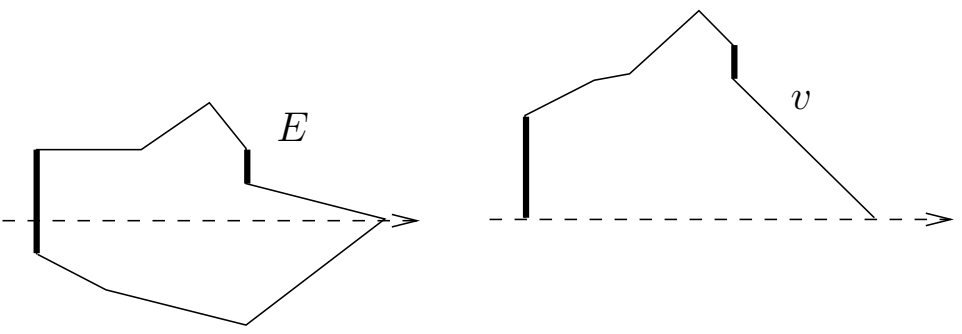

FIGURE 12. The continuity of $v(t)=\mathcal{H}^{n-1}\left(E \cap\left\{x_{1}=t\right\}\right)$ is related to the existence of parts of the reduced boundary of $E$ where $\nu_{E}=$ $\pm e_{1}$. 
A direct consequence of the Coarea Formula (7.1) is that the function $v$ has bounded variation on $\mathbb{R}$, and that, furthermore, as soon as the geometric condition

$$
\mathcal{H}^{n-1}\left(\left\{x \in \mathcal{F} E: \nu_{E}(x)= \pm e_{1}\right\}\right)=0,
$$

is fulfilled, it is in fact absolutely continuous on $\mathbb{R}$, with classical derivative

$$
v^{\prime}(t)=-\int_{\mathcal{F} E_{t}} \frac{\nu_{E} \cdot e_{1}}{\sqrt{1-\left|\nu_{E} \cdot e_{1}\right|^{2}}} d \mathcal{H}^{n-2}
$$

at a.e. $t \in \mathbb{R}$. We briefly prove this claim. In order to show that $v$ has bounded variation on $\mathbb{R}$, it suffices to show that

$$
\sup \left\{\int_{\mathbb{R}} \varphi^{\prime}(t) v(t) d t: \varphi \in C_{c}^{1}(\mathbb{R} ;[-1,1])\right\}<\infty .
$$

To this end, let us consider a sequence $\left\{\psi_{h}\right\}_{h \in \mathbb{N}} \in C_{c}^{1}\left(\mathbb{R}^{n-1} ;[0,1]\right)$, with $\psi_{h} \uparrow 1$, and define $f_{h}(x)=\varphi\left(x_{1}\right) \psi_{h}\left(x-x_{1} e_{1}\right)$. Then $f_{h} \in C_{c}^{1}\left(\mathbb{R}^{n}\right)$ and

$$
\begin{aligned}
\int_{\mathbb{R}} \varphi^{\prime} v & =\lim _{h \rightarrow \infty} \int_{\mathbb{R}} d t \int_{E_{t}} \varphi^{\prime}(t) \psi_{h}(z) d \mathcal{H}^{n-1}(z)=\lim _{h \rightarrow \infty} \int_{E} \frac{\partial f_{h}}{\partial x_{1}}(x) d x \\
& =\lim _{h \rightarrow \infty} \int_{\mathcal{F} E} f_{h}(x) \nu_{E}(x) \cdot e_{1} d \mathcal{H}^{n-1}(x)=\int_{\mathcal{F} E} \varphi\left(x_{1}\right) \nu_{E}(x) \cdot e_{1} d \mathcal{H}^{n-1}(x) .
\end{aligned}
$$

The absolute value of the right-hand side is bounded by $\int_{\mathcal{F} E}\left|\nu_{E} \cdot e_{1}\right| d \mathcal{H}^{n-1} \leq$ $\mathcal{H}^{n-1}(\mathcal{F} E)=P(E)$, thus $v$ has bounded variation. If we further assume the validity of (7.2), then $\sqrt{1-\left|\nu_{E} \cdot e_{1}\right|^{2}}>0$ at $\mathcal{H}^{n-1}$-a.e. point of $\mathcal{F} E$. By the Coarea Formula (17.1), we find

$$
\begin{aligned}
& \int_{\mathcal{F} E} \varphi\left(x_{1}\right) \nu_{E}(x) \cdot e_{1} d \mathcal{H}^{n-1}(x) \\
= & \int_{\mathcal{F} E} \varphi\left(x_{1}\right) \frac{\nu_{E}(x) \cdot e_{1}}{\sqrt{1-\left|\nu_{E}(x) \cdot e_{1}\right|^{2}}} \sqrt{1-\left|\nu_{E}(x) \cdot e_{1}\right|^{2}} d \mathcal{H}^{n-1}(x) \\
= & \int_{\mathbb{R}} \varphi(t) d t \int_{\mathcal{F} E_{t}} \frac{\nu_{E} \cdot e_{1}}{\sqrt{1-\left|\nu_{E} \cdot e_{1}\right|^{2}}} d \mathcal{H}^{n-2},
\end{aligned}
$$

and (7.3) follows. We are then in the position of proving the Schwarz inequality (5.4).

Proof of Theorem [5.1. Without loss of generality we may assume that $E$ has finite perimeter and consider its Schwartz symmetral $E^{*}$ with respect to the $x_{1}$-direction. As $\mathcal{H}^{n-1}(\mathcal{F} E)<\infty$, it can be

$$
\mathcal{H}^{n-1}\left(\left\{x \in \mathcal{F} E: \nu_{E}(x)= \pm \nu\right\}\right)>0
$$

for at most countably many $\nu \in S^{n-1}$. Thus, up to applying a rotation to $E$, we may also assume (7.2) is in force. In particular $\sqrt{1-\left|\nu_{E} \cdot e_{1}\right|^{2}}>0$ on $\mathcal{F} E$ up to a $\mathcal{H}^{n-1}$-negligible set. Therefore, by the Coarea Formula (7.1), by the Jensen 


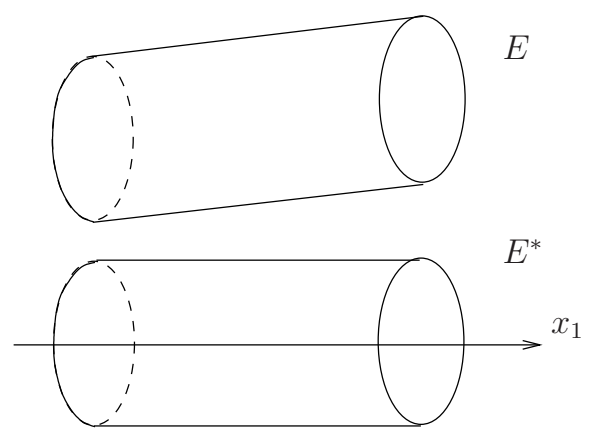

Figure 13. Here $P(E)>\int_{\mathbb{R}} \sqrt{p^{2}+\left(v^{\prime}\right)^{2}}=\int_{\mathbb{R}} \sqrt{p_{*}^{2}+\left(v^{\prime}\right)^{2}}=$ $P\left(E^{*}\right)$ : the inequality sign in (17.4) can be strict even if $v \in A C$, as long as $\nu_{E} \cdot e_{1}$ is not constant on each $E_{t}$.

inequality applied to $\Phi(z):=\sqrt{1+z^{2}}, z \in \mathbb{R}$, and by (7.3), we find

$$
\begin{aligned}
P(E) & =\mathcal{H}^{n-1}(\mathcal{F} E) \\
& =\int_{\mathcal{F} E} \frac{\sqrt{1-\left|\nu_{E} \cdot e_{1}\right|^{2}}}{\sqrt{1-\left|\nu_{E} \cdot e_{1}\right|^{2}}} d \mathcal{H}^{n-1}=\int_{\mathbb{R}} d t \int_{\mathcal{F} E_{t}} \frac{1}{\sqrt{1-\left|\nu_{E} \cdot e_{1}\right|^{2}}} d \mathcal{H}^{n-2} \\
& =\int_{\mathbb{R}} \int_{\mathcal{F} E_{t}} \Phi\left(\frac{\nu_{E} \cdot e_{1}}{\sqrt{1-\left|\nu_{E} \cdot e_{1}\right|^{2}}}\right) d \mathcal{H}^{n-2} \\
& \geq \int_{\mathbb{R}} \mathcal{H}^{n-2}\left(\mathcal{F} E_{t}\right) \Phi\left(\frac{1}{\mathcal{H}^{n-2}\left(\left(\mathcal{F} E_{t}\right)\right)} \int_{\mathcal{F} E_{t}} \frac{\nu_{E} \cdot e_{1}}{\sqrt{1-\left|\nu_{E} \cdot e_{1}\right|^{2}}}\right) d t \\
& =\int_{\mathbb{R}} \sqrt{\mathcal{H}^{n-2}\left(\mathcal{F} E_{t}\right)^{2}+v^{\prime}(t)^{2}} d t .
\end{aligned}
$$

Therefore, we have proved the following lower bound for the perimeter of $E$ under the geometric assumption (7.2):

$$
P(E) \geq \int_{\mathbb{R}} \sqrt{\mathcal{H}^{n-2}\left(\mathcal{F} E_{t}\right)^{2}+v^{\prime}(t)^{2}} d t .
$$

The same argument applies to $E^{*}$ (in particular, one sees that (7.2) holds for $E^{*}$ also), and in fact in this case equality holds, as $\nu_{E^{*}} \cdot e_{1}$ has to be constant on $\mathcal{F} E_{t}^{*}$, thanks to the symmetry of $E^{*}$. Therefore,

$$
P\left(E^{*}\right)=\int_{\mathbb{R}} \sqrt{\mathcal{H}^{n-2}\left(\mathcal{F} E_{t}^{*}\right)^{2}+v^{\prime}(t)^{2}} d t .
$$

Now recall that $\left(E^{*}\right)_{t}$ is an $(n-1)$-dimensional ball with the same $\mathcal{H}^{n-1}$-measure of $E_{t}$; thus, by the isoperimetric inequality in $\mathbb{R}^{n-1}$,

$$
\mathcal{H}^{n-2}\left(\mathcal{F} E_{t}\right) \geq \mathcal{H}^{n-2}\left(\mathcal{F} E_{t}^{*}\right) .
$$

We conclude that $P(E) \geq P\left(E^{*}\right)$.

7.2. Proof of Theorem 1.1. Without loss of generality we can assume that $|E|=$ $|B|$ and that $\delta(E) \leq \delta(n)$. By Theorems 4.1 and 4.4, we can directly assume that 
$E \subset[0, L(n)]^{n}$ and that $E$ is indecomposable. Up to a rotation, we can also ask that

$$
\mathcal{H}^{n-1}\left(\left\{x \in \mathcal{F} E: \nu_{E}(x)= \pm e_{k}, k=1, \ldots, n\right\}\right)=0 .
$$

Then we apply Theorem 6.1 to $E$, using hyperplanes parallel to the coordinate axes. The resulting set is constructed by reflecting $n$-times a certain subset of $E$ with respect to the coordinate directions; therefore, it still satisfies the geometric condition (7.5), and it is contained into $[-L(n), L(n)]^{n}$.

Summarizing, we can assume without loss of generality that $|E|=|B|, \delta(E) \leq$ $\delta(n), E \subset[-L(n), L(n)]^{n}, E$ is indecomposable, $E$ is $n$-symmetric with respect to the coordinate axes, and (7.5) is in force. Under these conditions, we are going to show that

$$
|E \Delta B| \leq C(n) \sqrt{\delta(E)} .
$$

The theorem then follows, as $A(E) \leq|B|^{-1}|E \Delta B|$.

Let us now consider the stripes $S_{1}=\left\{x:\left|x_{1}\right|<\sqrt{2} / 2\right\}$ and $S_{2}=\left\{x:\left|x_{2}\right|<\right.$ $\sqrt{2} / 2\}$. As $B \subset S_{1} \cup S_{2}$, we have that one between $\left|(B \backslash E) \cap S_{1}\right|$ and $\left|(B \backslash E) \cap S_{2}\right|$ is at least $|B \backslash E| / 2$. Up to a rotation, we can assume this happens in the former case. As $|E|=|B|$, we have $|E \Delta B|=2|B \backslash E|$; therefore,

$$
|E \Delta B| \leq 4\left|(B \backslash E) \cap S_{1}\right| \text {. }
$$

Now we let $E^{*}$ be the Schwarz symmetral of $E$ with respect to the $x_{1}$-axis. Note that $E^{*}$, further than being axially symmetric around the $x_{1}$-axis, is also symmetric with respect to the coordinate hyperplanes. We use $E^{*}$ as intermediate comparison object between $E$ and $B$,

$$
\left|(B \backslash E) \cap S_{1}\right| \leq\left|\left(E^{*} \backslash E\right) \cap S_{1}\right|+\left|\left(B \backslash E^{*}\right) \cap S_{1}\right|,
$$

and then pause to show that

$$
\begin{aligned}
& \left|\left(E^{*} \Delta E\right) \cap S_{1}\right| \leq C(n) \sqrt{\delta(E)}, \\
& \left|\left(B \Delta E^{*}\right) \cap S_{1}\right| \leq C(n) \sqrt{\delta\left(E^{*}\right)} .
\end{aligned}
$$

Once we have proved these two estimates, as $P\left(E^{*}\right) \leq P(E)$ and therefore $\delta\left(E^{*}\right) \leq$ $\delta(E)$, (7.6) shall follow from (7.7) and (7.8).

Step one: A lower bound on $v$. This is a small but crucial technical step. Let $w(t)=\mathcal{H}^{n-1}\left(B \cap\left\{x_{1}=t\right\}\right)$, and let $c_{0}=c_{0}(n)=w(\lambda)$ for some fixed $\lambda \in(\sqrt{2} / 2,1)$. We claim that, provided $\delta(n)$ is small enough, we have

$$
\inf \{v(t): t \in I\} \geq \frac{c_{0}}{3}, \quad I=\left(-\frac{\sqrt{2}}{2}, \frac{\sqrt{2}}{2}\right) .
$$

Otherwise let $t_{0} \in I$ be such that $v\left(t_{0}\right)<c_{0} / 3$, and consider $\left(t_{1}, t_{2}\right)$ defined as the smallest interval containing $t_{0}$, contained in $(-\lambda, \lambda)$, and such that

$$
v(t) \leq \frac{c_{0}}{2}, \quad \forall t \in\left(t_{1}, t_{2}\right) .
$$

Note that $t_{1}<t_{0}<t_{2}$, and that

$$
\frac{c_{0}}{2}\left(t_{2}-t_{1}\right) \leq \int_{\mathbb{R}}|w-v|=\left|E^{*} \Delta B\right| \leq 3|B| A\left(E^{*}\right),
$$


where in the last inequality we used Lemma 5.2 and the fact that $E^{*}$ is $n$-symmetric. Thanks to Theorem 4.3, as $\delta\left(E^{*}\right) \leq \delta(E) \leq \delta(n)$, we have

$$
t_{2}-t_{1} \leq \omega(\delta(n)),
$$

for a continuous function $\omega:[0, \infty) \rightarrow[0, \infty)$, independent of $E^{*}$, and such that $\omega\left(0^{+}\right)=0$. In particular, as $t_{0} \in I$, both $t_{2}-t_{0}$ and $t_{0}-t_{1}$ can be assumed to be small enough in order to have $-\lambda<t_{1}$ and $t_{2}<\lambda$, and therefore

$$
v\left(t_{1}\right)=v\left(t_{2}\right)=\frac{c_{0}}{2} .
$$

Now let $F$ be the axially symmetric set such that $\mathcal{H}^{n-1}\left(F \cap\left\{x_{1}=t\right\}\right)$ equals $v(t)$ outside $\left(t_{1}, t_{2}\right)$, and it takes the constant value $v\left(t_{1}\right)=v\left(t_{2}\right)=c_{0} / 2$ on $\left(t_{1}, t_{2}\right)$. Let also $S=\left(t_{1}, t_{2}\right) \times \mathbb{R}^{n-1}$. Then

$$
P\left(E^{*}\right)=P(F)-P(F \mid S)+P\left(E^{*} \mid S\right) .
$$

Concerning the third term, by arguing as in the proof of (7.4),

$$
\begin{aligned}
P\left(E^{*} \mid S\right) & \geq \int_{t_{1}}^{t_{2}} \sqrt{\mathcal{H}^{n-1}\left(\mathcal{F} E_{t}^{*}\right)^{2}+v^{\prime}(t)^{2}} d t \geq \int_{t_{1}}^{t_{2}}\left|v^{\prime}\right| \\
& \geq\left|v\left(t_{1}\right)-v\left(t_{0}\right)\right|+\left|v\left(t_{0}\right)-v\left(t_{1}\right)\right|=\frac{c_{0}}{3} .
\end{aligned}
$$

The second term is controlled elementarily, as clearly

$$
P(F \mid S)=c(n)\left|t_{1}-t_{2}\right| \text {. }
$$

In the end, by the isoperimetric inequality,

$$
P(F) \geq n|B|^{1 / n}|F|^{1 / n^{\prime}} \geq n|B|^{1 / n}\left(\left|E^{*}\right|-C(n)\left|t_{1}-t_{2}\right|\right)^{1 / n^{\prime}}
$$

therefore, we conclude that

$$
P\left(E^{*}\right) \geq n|B|^{1 / n}\left|E^{*}\right|^{1 / n^{\prime}}+\frac{c_{0}}{3}-\omega(\delta(n)),
$$

which leads to a contradiction as soon as $\delta(n)$ is small enough.

Step two: Estimate for $\left|\left(E^{*} \Delta B\right) \cap S_{1}\right|$. We are going to prove (7.10). This inequality is implied by Hall's theorem, and an alternative proof is found in [FMP1]. Both proofs are somehow based on a parametrization of $B$ in terms of $E$ through the function $\tau$ defined by

$$
\left|E^{*} \cap\left\{x_{1}<t\right\}\right|=\left|E \cap\left\{x_{1}<t\right\}\right|=\left|B \cap\left\{x_{1}<\tau(t)\right\}\right| .
$$

We present here a variant of these arguments, still based on the use of the function $\tau$, and inspired by Gromov's proof of the isoperimetric inequality MS, CNV, FiMP.

Since $E$ is indecomposable and $v$ is continuous, then $\{t \in \mathbb{R}: v(t)>0\}$ is an interval. By symmetry, $\{v>0\}=(-M, M)$. Note that, thanks to the lower bound (7.11), we have $M>\sqrt{2} / 2$, i.e. $I \subset(-M, M)$. It turns out that (7.13) defines $\tau:(-M, M) \rightarrow(-1,1)$. By construction

$$
\int_{-\infty}^{t} v(s) d s=\int_{-\infty}^{\tau(t)} w(s) d s .
$$

It is immediately proved that $\tau \in C^{1}((-M, M))$ with $\tau^{\prime}>0$ given by

$$
\tau^{\prime}(t)=\frac{v(t)}{w(\tau(t))}, \quad \forall t \in(-M, M) .
$$


As $v$ is absolutely continuous, we also find that, at a.e. $t \in(-M, M)$, there exists $\tau^{\prime \prime}(t)$, and it equals

$$
\tau^{\prime \prime}(t)=\frac{v^{\prime}(t) w(\tau(t))^{2}-w^{\prime}(\tau(t)) v(t)^{2}}{w(\tau(t))^{3}}
$$

Thus $\tau \in W_{l o c}^{2,1}(-M, M)$. As $I \subset(-M, M)$, and as $w$ is bounded on $[-1,1]$ and uniformly Lipschitz on $I$, we have that

$$
\begin{aligned}
\left|\left(E^{*} \Delta B\right) \cap S_{1}\right| & =\int_{I}|v-w|=\int_{I}\left|w(\tau(t)) \tau^{\prime}(t)-w(t)\right| d t \\
& \leq \int_{I} w(\tau(t))\left|\tau^{\prime}(t)-1\right| d t+\int_{I}|w(\tau(t))-w(t)| d t \\
& \leq C(n) \int_{I}\left|\tau^{\prime}(t)-1\right|+|\tau(t)-t| d t \leq C(n) \int_{I}\left|\tau^{\prime}-1\right|,
\end{aligned}
$$

where in the last step we have used the fact that $\tau(0)=0$ to deduce $|\tau(t)-t| \leq$ $\int_{I}\left|\tau^{\prime}-1\right|$ whenever $t \in I$. Therefore, in order to conclude the proof of Step two, we need an estimate for $\left\|\tau^{\prime}-1\right\|_{L^{1}(I)}$.

Let us consider the deformation $T:(-M, M) \times \mathbb{R}^{n-1} \rightarrow \mathbb{R}^{n}$, defined by

$$
T(x)=\tau\left(x_{1}\right) e_{1}+\sum_{i=2}^{n}\left(\frac{w\left(\tau\left(x_{1}\right)\right)}{v\left(x_{1}\right)}\right)^{1 /(n-1)} x_{i} e_{i} .
$$

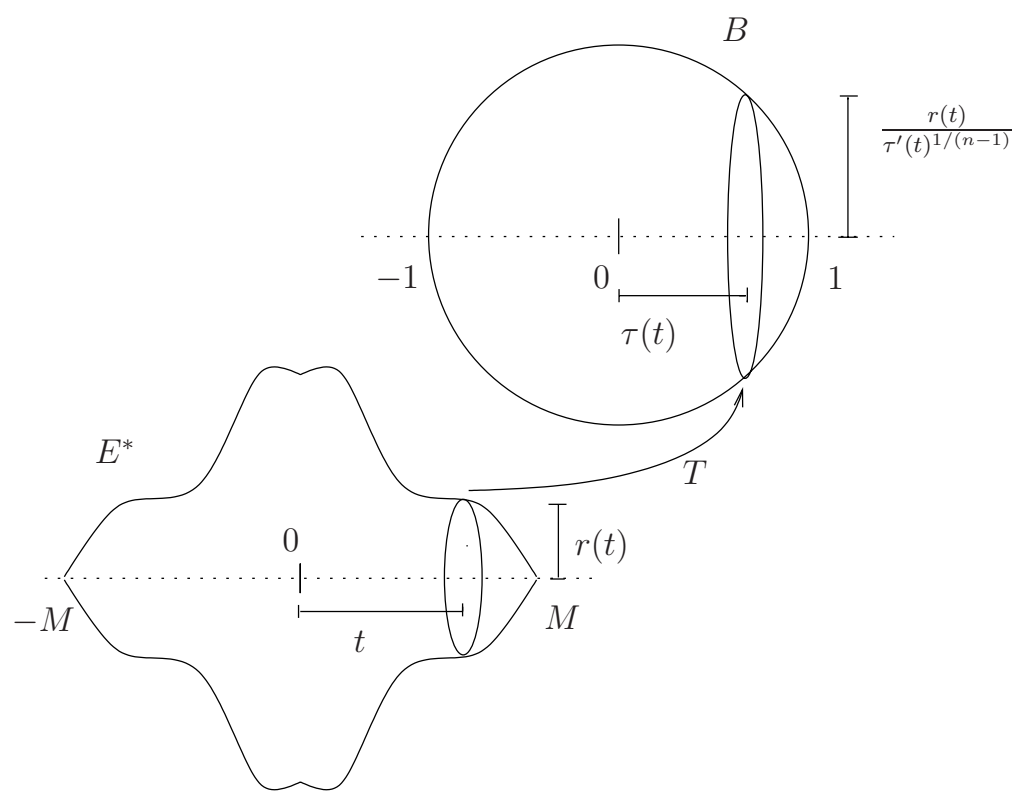

Figure 14. The deformation $T ; E^{*} \cap\left\{x_{1}=t\right\}$ is sent into $B \cap$ $\left\{x_{1}=\tau(t)\right\}$. 
Note that $T\left(E^{*} \cap\left\{x_{1}=t\right\}\right)=B \cap\left\{x_{1}=\tau(t)\right\}$ for every $t \in\{v>0\}$, therefore, $T\left(E^{*}\right)=B$. By (7.15) we can alternatively write

$$
T(x)=\tau\left(x_{1}\right) e_{1}+\sum_{i=2}^{n} \frac{1}{\tau^{\prime}\left(x_{1}\right)^{1 /(n-1)}} x_{i} e_{i}, \quad \forall x \in(-M, M) \times \mathbb{R}^{n-1} .
$$

At a.e. $x \in(-M, M) \times \mathbb{R}^{n-1}$, the gradient of $T$ is the tensor

$\nabla T(x)=\tau^{\prime}\left(x_{1}\right) e_{1} \otimes e_{1}+\sum_{i=2}^{n} \frac{1}{\tau^{\prime}\left(x_{1}\right)^{1 /(n-1)}} e_{i} \otimes e_{i}+\left(\sum_{i=2}^{n} \frac{\partial\left(\tau^{\prime}\left(x_{1}\right)^{1 /(1-n)}\right)}{\partial x_{1}} x_{i} e_{i}\right) \otimes e_{1}$,

and $\nabla T(x)$ has at most two distinct eigenvalues: $\tau^{\prime}\left(x_{1}\right)$ in the direction $e_{1}$, and $\left(1 / \tau^{\prime}\left(x_{1}\right)\right)^{1 /(n-1)}$ isotropically on $e_{1}^{\perp}$. Thus we find

$$
\operatorname{div} T(x)=\tau^{\prime}\left(x_{1}\right)+(n-1) \frac{1}{\tau^{\prime}\left(x_{1}\right)^{1 /(n-1)}} .
$$

By Young's inequality

$$
\frac{\operatorname{div} T(x)}{n}=\frac{1}{n}\left(\tau^{\prime}\left(x_{1}\right)^{1 / n}\right)^{n}+\frac{1}{n^{\prime}}\left(\frac{1}{\tau^{\prime}\left(x_{1}\right)^{1 / n}}\right)^{n^{\prime}} \geq 1 .
$$

As $T(x) \in B$, by the Divergence Theorem (this is legitimate, as explained in the appendix)

$P\left(E^{*}\right)=\mathcal{H}^{n-1}\left(\mathcal{F} E^{*}\right) \geq \int_{\mathcal{F} E^{*}} T \cdot \nu_{E} d \mathcal{H}^{n-1}=\int_{E^{*}} \operatorname{div} T \geq n\left|E^{*}\right|=n|B|^{1 / n}\left|E^{*}\right|^{1 / n^{\prime}} ;$

therefore,

$$
|B| \delta\left(E^{*}\right) \geq \int_{E^{*}}\left\{\frac{\operatorname{div} T}{n}-1\right\} \geq \int_{-M}^{M}\left\{\frac{1}{n} \tau^{\prime}(t)+\frac{(n-1)}{n} \frac{1}{\tau^{\prime}(t)^{1 /(n-1)}}-1\right\} v(t) d t .
$$

Then we set $\sigma=\left(\tau^{\prime}\right)^{1 /(n-1)}$ and notice that (see Figure 15)

$$
C(n) \delta\left(E^{*}\right) \geq \int_{-M}^{M} \frac{v}{\sigma}\left\{\frac{\sigma^{n}}{n}+\frac{1}{n^{\prime}}-\sigma\right\} d t \geq c(n) \int_{-M}^{M} \frac{v}{\sigma}(\sigma-1)^{2} d t .
$$

By the Hölder inequality,

$$
\begin{aligned}
& \int_{-M}^{M} v|\sigma-1| \leq C(n) \sqrt{\int_{-M}^{M} \frac{v}{\sigma}(\sigma-1)^{2}} \sqrt{\int_{-M}^{M} v \sigma} \leq C(n) \sqrt{\delta\left(E^{*}\right)}, \\
& \frac{\sigma^{n}}{n}+\frac{1}{n^{\prime}}-\sigma
\end{aligned}
$$

Figure 15 
where

$$
\int_{-M}^{M} v \sigma \leq\left(\int_{-M}^{M} \tau^{\prime}\right)^{1 /(n-1)}\left(\int_{-M}^{M} v^{(n-1) /(n-2)}\right)^{(n-2) /(n-1)} \leq C(n),
$$

as $\int_{-M}^{M} \tau^{\prime}=2$ and $\|v\|_{L^{1}(\mathbb{R})}=|E|,\|v\|_{L^{\infty}(\mathbb{R})} \leq(2 L(n))^{n-1}$. By the lower bound on $v$ (7.11), we deduce from (7.17) that

$$
C(n) \sqrt{\delta\left(E^{*}\right)} \geq \int_{I}|\sigma-1|=\int_{I}\left|\left(\tau^{\prime}\right)^{1 /(n-1)}-1\right| \geq c(n) \int_{I}\left|\tau^{\prime}-1\right|,
$$

as soon as we show that $\left\|\tau^{\prime}\right\|_{L^{\infty}(I)} \leq C(n)$. By 7.15 and thanks to the uniform upper bound on $v$, it is enough to show that for a constant $\lambda(n) \in(0,1)$ we have

$$
\tau(t) \in[-\lambda(n), \lambda(n)], \quad \forall t \in I .
$$

Indeed, on the one hand

$\left|E^{*} \cap\left\{x_{1}>\sqrt{2} / 2\right\}\right| \geq\left|B \cap\left\{x_{1}>\sqrt{2} / 2\right\}\right|-\left|E^{*} \Delta B\right| \geq c_{1}(n)-3|B| A\left(E^{*}\right) \geq c_{2}(n)$.

On the other hand

$$
\left|E^{*} \cap\left\{x_{1}>\sqrt{2} / 2\right\}\right|=\left|B \cap\left\{x_{1}>\tau(\sqrt{2} / 2)\right\}\right| \leq \omega_{n-1}(1-\tau(\sqrt{2} / 2)) .
$$

Thus $1-\tau(\sqrt{2} / 2) \geq c(n)$, and we have proved (7.18).

Step three. We show that if the theorem is valid in dimension $n-1$, then (7.9) holds true. In particular, if the theorem holds in dimension $n-1$, then it holds in dimension $n$.

Let us recall that

$$
P(E) \geq \int_{\mathbb{R}} \sqrt{p(t)^{2}+v^{\prime}(t)^{2}} d t, \quad P\left(E^{*}\right)=\int_{\mathbb{R}} \sqrt{p_{*}(t)^{2}+v^{\prime}(t)^{2}} d t, \quad p(t) \geq p_{*}(t),
$$

as shown in the proof of (5.4); note that here we have set for brevity

$$
p(t)=\mathcal{H}^{n-2}\left(\mathcal{F} E_{t}\right), \quad p_{*}(t)=\mathcal{H}^{n-2}\left(\mathcal{F} E_{t}^{*}\right) .
$$

As we are assuming (4.1) in dimension $n-1$, we know that, for a.e. $t \in(-M, M)$,

$$
\text { Fraenkel asymmetry of } E_{t} \text { in } \mathbb{R}^{n-1} \leq C(n) \sqrt{\frac{p(t)}{p_{*}(t)}-1} \text {. }
$$

As $E$ is symmetric by reflection with respect to the coordinate axes, the section $E_{t}$ turns out to be $(n-1)$-symmetric in $\mathbb{R}^{n-1}$ with respect to the coordinate axes too. In particular, up to the multiplicative factor 3, we know that the Fraenkel asymmetry of $E_{t}$ in $\mathbb{R}^{n-1}$ is in fact equivalent to the distance of $E_{t}$ from $E_{t}^{*}$, the $(n-1)$-dimensional ball with the same $\mathcal{H}^{n-1}$-dimensional measure as $E_{t}$, centered at $t e_{1}$, and oriented by $e_{1}$. We come to the estimate

$$
\frac{\mathcal{H}^{n-1}\left(E_{t} \Delta E_{t}^{*}\right)}{v(t)} \leq C(n) \sqrt{\frac{p(t)-p_{*}(t)}{p_{*}(t)}} .
$$

We are now in the position to prove (7.9). On the one hand,

$$
\left|\left(E^{*} \Delta E\right) \cap S_{1}\right|=\int_{I} \mathcal{H}^{n-1}\left(E_{t} \Delta E_{t}^{*}\right) d t \leq C(n) \int_{I} \sqrt{\frac{p(t)-p_{*}(t)}{p_{*}(t)}} d t,
$$


as $\|v\|_{L^{\infty}(\mathbb{R})} \leq(2 L(n))^{n-1}$. On the other hand,

$$
\delta(E)=\frac{P(E)}{P(B)}-1=\frac{P(E)-P\left(E^{*}\right)}{P(B)}+\delta\left(E^{*}\right),
$$

so that

$$
\begin{aligned}
P(B) \delta(E) & \geq P(E)-P\left(E^{*}\right) \geq \int_{\mathbb{R}} \sqrt{p^{2}+\left(v^{\prime}\right)^{2}}-\sqrt{p_{*}^{2}+\left(v^{\prime}\right)^{2}} \\
& =\int_{\mathbb{R}} \frac{p^{2}-p_{*}^{2}}{\sqrt{p^{2}+\left(v^{\prime}\right)^{2}}+\sqrt{p_{*}^{2}+\left(v^{\prime}\right)^{2}}} .
\end{aligned}
$$

By the Hölder inequality

$$
\begin{aligned}
\int_{\mathbb{R}} \sqrt{p^{2}-p_{*}^{2}} & \leq \sqrt{\int_{\mathbb{R}} \frac{p^{2}-p_{*}^{2}}{\sqrt{p^{2}+\left(v^{\prime}\right)^{2}}+\sqrt{p_{*}^{2}+\left(v^{\prime}\right)^{2}}}} \sqrt{\int_{\mathbb{R}} \sqrt{p^{2}+\left(v^{\prime}\right)^{2}}+\sqrt{p_{*}^{2}+\left(v^{\prime}\right)^{2}}} \\
& \leq \sqrt{P(B) \delta(E)} \sqrt{P(E)+P\left(E^{*}\right)}
\end{aligned}
$$

As $P(E)+P\left(E^{*}\right) \leq 2 P(E) \leq 2 P(B)(1+\delta(E)) \leq C(n)$, we have

$$
C(n) \sqrt{\delta(E)} \geq \int_{\mathbb{R}} \sqrt{p^{2}-p_{*}^{2}} \geq \int_{\mathbb{R}} p_{*} \sqrt{\frac{p}{p_{*}}-1} .
$$

Therefore, thanks to (7.19),

$$
C(n) \sqrt{\delta(E)} \geq \inf \left\{p_{*}(t): t \in I\right\}\left|\left(E^{*} \Delta E\right) \cap S_{1}\right| \geq c(n)\left|\left(E^{*} \Delta E\right) \cap S_{1}\right|,
$$

where we have used (7.11) and the fact that $p_{*}(t)$ is a power of $v(t)$. The proof of Step three is achieved.

Step four. We remark that the theorem being trivially valid in dimension one, the statement of Step three implies the thesis via an induction argument. This remark concludes the proof of Theorem 1.1

\section{APPENDIX}

In this brief appendix we provide a justification of (7.16). Let $0<N<M$ be fixed. We claim that

$$
T \in W^{1,1}\left(E^{*} \cap\left[(-N, N) \times \mathbb{R}^{n-1}\right] ; B\right) .
$$

To see this, we start by noticing that

$$
\frac{\partial T^{(1)}}{\partial x_{1}}(x)=\tau^{\prime}\left(x_{1}\right)
$$

with $\tau^{\prime}$ bounded on $(-N, N)$. Moreover,

$$
\frac{\partial T^{(i)}}{\partial x_{i}}(x)=\frac{1}{\tau^{\prime}\left(x_{1}\right)^{1 /(n-1)}}=\left(\frac{w\left(\tau\left(x_{1}\right)\right)}{v\left(x_{1}\right)}\right)^{1 /(n-1)} .
$$

As $w$ is bounded from above on $\mathbb{R}$ and $v$ is locally bounded from below on $(-M, M)$ (recall that $v$ is continuous and $(-M, M)=\{v>0\}$ ), it turns out that this partial derivative is bounded on $E^{*} \cap\left[(-N, N) \times \mathbb{R}^{n-1}\right]$. In particular,

$$
\operatorname{div} T \in L^{\infty}\left(E^{*} \cap\left[(-N, N) \times \mathbb{R}^{n-1}\right]\right) .
$$

In conclusion we have to notice that

$$
\frac{\partial T^{(i)}}{\partial x_{1}}(x)=\frac{-1}{n-1} \frac{\tau^{\prime \prime}\left(x_{1}\right)}{\tau^{\prime}\left(x_{1}\right)^{n^{\prime}}} .
$$


As seen, $1 / \tau^{\prime}$ is locally bounded on $(-N, N)$, while $\tau^{\prime \prime} \in L_{l o c}^{1}(-M, M)$, as we have already noticed in Step two of the proof of Theorem 1.1. Thus this partial derivative belongs to $L^{1}\left(E^{*} \cap\left[(-N, N) \times \mathbb{R}^{n-1}\right]\right)$, and (A.1) is proved. Now let $\nu$ be the outer unit normal vector to $E^{*} \cap\left[(-N, N) \times \mathbb{R}^{n-1}\right]$, then

$$
\begin{aligned}
& n\left|E^{*} \cap\left[(-N, N) \times \mathbb{R}^{n-1}\right]\right|=\int_{E^{*} \cap\left[(-N, N) \times \mathbb{R}^{n-1}\right]} \operatorname{div} T \\
= & \int_{\mathcal{F}\left(E^{*} \cap\left[(-N, N) \times \mathbb{R}^{n-1}\right]\right)} T \cdot \nu d \mathcal{H}^{n-1} \leq \mathcal{H}^{n-1}\left(\mathcal{F} E^{*}\right)+2 v(N) .
\end{aligned}
$$

By continuity of $v$, as $N \rightarrow M^{-}, v(N) \rightarrow 0$, and we recover

$$
\mathcal{H}^{n-1}\left(\mathcal{F} E^{*}\right) \geq \int_{E^{*}} \operatorname{div} T \geq n\left|E^{*}\right|
$$

as needed in Step two.

\section{ACKNOWLEDGMENTS}

As mentioned in the Introduction, this work covers the material taught in the first part of a Ph.D. course held by the author at the Università di Napoli "Federico II" during May 2007. It is a pleasure to thank Nicola Fusco for the invitation to hold the above-mentioned course, and the students and the colleagues attending the lectures for their interest in this subject. Most importantly, I wish to acknowledge the influence of Nicola Fusco and Aldo Pratelli in my understanding of these problems. Alessio Figalli is also thanked for several interesting remarks on a preliminary version of this paper.

While working on this project, I have been partially supported by the GNAMPA through the 2007 research project Disuguaglianze geometrico-funzionali in forma ottimale e quantitativa.

\section{About the Author}

Francesco Maggi is a researcher at the Università di Firenze. He has also held positions at the MPI-MPS Leipzig and at the Universitet Duisburg-Essen. He has visited various universities and institutes in France, Germany, Italy, Switerzerland, and the United States.

\section{REFERENCES}

[AFN] A. Alvino, V. Ferone, C. Nitsch, The quantitative isoperimetric inequality for convex domains in the plane, preprint.

[AFP] L. Ambrosio, N. Fusco, D. Pallara, Functions of bounded variation and free discontinuity problems. Oxford Mathematical Monographs. The Clarendon Press, Oxford University Press, New York, 2000, xviii+434 pp. MR 1857292 (2003a:49002)

[Be] F. Bernstein, Über die isoperimetriche Eigenschaft des Kreises auf der Kugeloberflache und in der Ebene, Math. Ann., 60 (1905), 117-136. MR.1511289

[BE] G. Bianchi, H. Egnell, A note on the Sobolev inequality, J. Funct. Anal. 100 (1991), no. 1, 18-24. MR1124290 (92i:46033)

[Bl] G.A. Bliss, An integral inequality, J. London Math. Soc. 5 (1930), 40-46.

[Bo] T. Bonnesen, Über die isoperimetrische Defizit ebener Figuren, Math. Ann. 91 (1924), 252-268. MR 1512192

[BL] H. Brezis, E. H. Lieb, Sobolev inequalities with remainder terms, J. Funct. Anal. 62 (1985), no. 1, 73-86. MR790771 (86i:46033)

[Ci1] A. Cianchi, A quantitative Sobolev inequality in BV, J. Funct. Anal. 237 (2006), no. 2, 466-481. MR2230346 (2007b:46053) 
[Ci2] A. Cianchi, Sharp Sobolev-Morrey inequalities and the distance from extremals, to appear in Trans. Amer. Math. Soc.

[CCF] M. Chlebík, A. Cianchi, N. Fusco, The perimeter inequality under Steiner symmetrization: cases of equality. Ann. of Math. (2) 162 (2005), no. 1, 525-555. MR2178968 (2006m:49032)

[CEFT] A. Cianchi, L. Esposito, N. Fusco, C. Trombetti, A quantitative Pólya-Szegö principle, to appear in J. Reine Angew. Math.

[CFMP1] A. Cianchi, N. Fusco, F. Maggi, A. Pratelli, The sharp Sobolev inequality in quantitative form, submitted paper.

[CFMP2] A. Cianchi, N. Fusco, F. Maggi, A. Pratelli, On the isoperimetric deficit in the Gauss space, submitted paper.

[CNV] D. Cordero-Erausquin, B. Nazaret, C. Villani, A mass-transportation approach to sharp Sobolev and Gagliardo-Nirenberg inequalities, Adv. Math. 182 (2004), no. 2, 307-332. MR2032031 (2005b:26023)

[DG1] E. De Giorgi, Su una teoria generale della misura $(r-1)$-dimensionale in uno spazio ad $r$ dimensioni. (Italian) Ann. Mat. Pura Appl. (4) 36 (1954), 191-213. MR0062214 $(15: 945 \mathrm{~d})$

[DG2] E. De Giorgi, Nuovi teoremi relativi alle misure $(r-1)$-dimensionali in uno spazio ad $r$ dimensioni. (Italian) Ricerche Mat. 4 (1955), 95-113. MR0074499 (17:596a)

[DG3] E. De Giorgi, Sulla proprietà isoperimetrica dell'ipersfera, nella classe degli insiemi aventi frontiera orientata di misura finita. (Italian) Atti Accad. Naz. Lincei. Mem. Cl. Sci. Fis. Mat. Nat. Sez. I (8) 5 (1958), 33-44. MR0098331(20:4792)

[EFT] L. Esposito, N. Fusco, C. Trombetti, A quantitative version of the isoperimetric inequality: the anisotropic case. Ann. Sci. Norm. Super. Pisa Cl. Sci. (5) 4 (2005), no. 4, 619-651. MR2207737 (2006k:52013)

[EG] L. C. Evans, R. F. Gariepy, Measure theory and fine properties of functions. Studies in Advanced Mathematics. CRC Press, Boca Raton, FL, 1992, viii+268 pp. MR 1158660 (93f:28001)

[FiMP] A. Figalli, F. Maggi, A. Pratelli, A mass transportation approach to isoperimetric type inequalities in quantitative form, in preparation.

[FR] W. H. Fleming, R. Rishel, An integral formula for total gradient variation. Arch. Math. 11 (1960), 218-222. MR0114892(22:5710)

[FM] I. Fonseca, S. Müller, A uniqueness proof for the Wulff theorem. Proc. Roy. Soc. Edinburgh Sect. A 119 (1991), no. 1-2, 125-136. MR1130601 (93c:49026)

[Fu] B. Fuglede, Stability in the isoperimetric problem for convex or nearly spherical domains in $\mathbb{R}^{n}$, Trans. Amer. Math. Soc. 314 (1989), 619-638. MR942426 (89m:52016)

[Fs] N. Fusco, The classical isoperimetric theorem, Rend. Acad. Sci. Fis. Mat. Napoli (4) 71 (2004), 63-107. MR2147710 (2006a:49069)

[FMP1] N. Fusco, F. Maggi, A. Pratelli, The sharp quantitative isoperimetric inequality, to appear in Ann. of Math.

[FMP2] N. Fusco, F. Maggi, A. Pratelli, The sharp quantitative Sobolev inequality for functions of bounded variation. J. Funct. Anal. 244 (2007), no. 1, 315-341. MR2294486

[FMP3] N. Fusco, F. Maggi, A. Pratelli, Stability estimates for certain Faber-Krahn, isocapacitary and Cheeger inequalities, submitted paper.

[Ha] R. R. Hall, A quantitative isoperimetric inequality in $n$-dimensional space, J. Reine Angew. Math. 428 (1992), 161-176. MR.1166511 (93d:51041)

[HHW] R. R. Hall, W. K. Hayman, A. W. Weitsman, On asymmetry and capacity, J. d'Analyse Math. 56 (1991), 87-123. MR:1243100 (95h:31004)

[Ka] B. Kawohl, Rearrangements and convexity of level sets in PDE, Lecture Notes in Math. 1150, Springer-Verlag, Berlin, 1985. MR810619 (87a:35001)

[MS] V. D. Milman, G. Schechtman, Asymptotic theory of finite-dimensional normed spaces. With an appendix by M. Gromov. Lecture Notes in Mathematics, 1200. Springer-Verlag, Berlin, 1986, viii+156 pp. MR856576 (87m:46038)

[Os1] R. Osserman, Bonnesen-style isoperimetric inequalities, Amer. Math. Monthly 86 (1979), 1-29. MR519520 (80h:52013)

[Os2] R. Osserman, The isoperimetric inequality, Bull. Amer. Math. Soc. 84 (1978), 11821238. MR $0500557(58: 18161)$ 
[PS] G. Pólya, G. Szegö, Isoperimetric inequalities in mathematical physics. Annals of Mathematics Studies, no. 27, Princeton University Press, Princeton, NJ, 1951. MR0043486 $(13: 270 \mathrm{~d})$

[Ta] G. Talenti, The standard isoperimetric theorem. Handbook of convex geometry, Vol. A, B, 73-123, North-Holland, Amsterdam, 1993. MR,1242977(94h:49065)

Dipartimento di Matematica, Università di Firenze, viale Morgagni 67/A, 50134 Firenze, Italy

E-mail address: maggi@math.unifi.it 